Provided for non-commercial research and education use. Not for reproduction, distribution or commercial use.

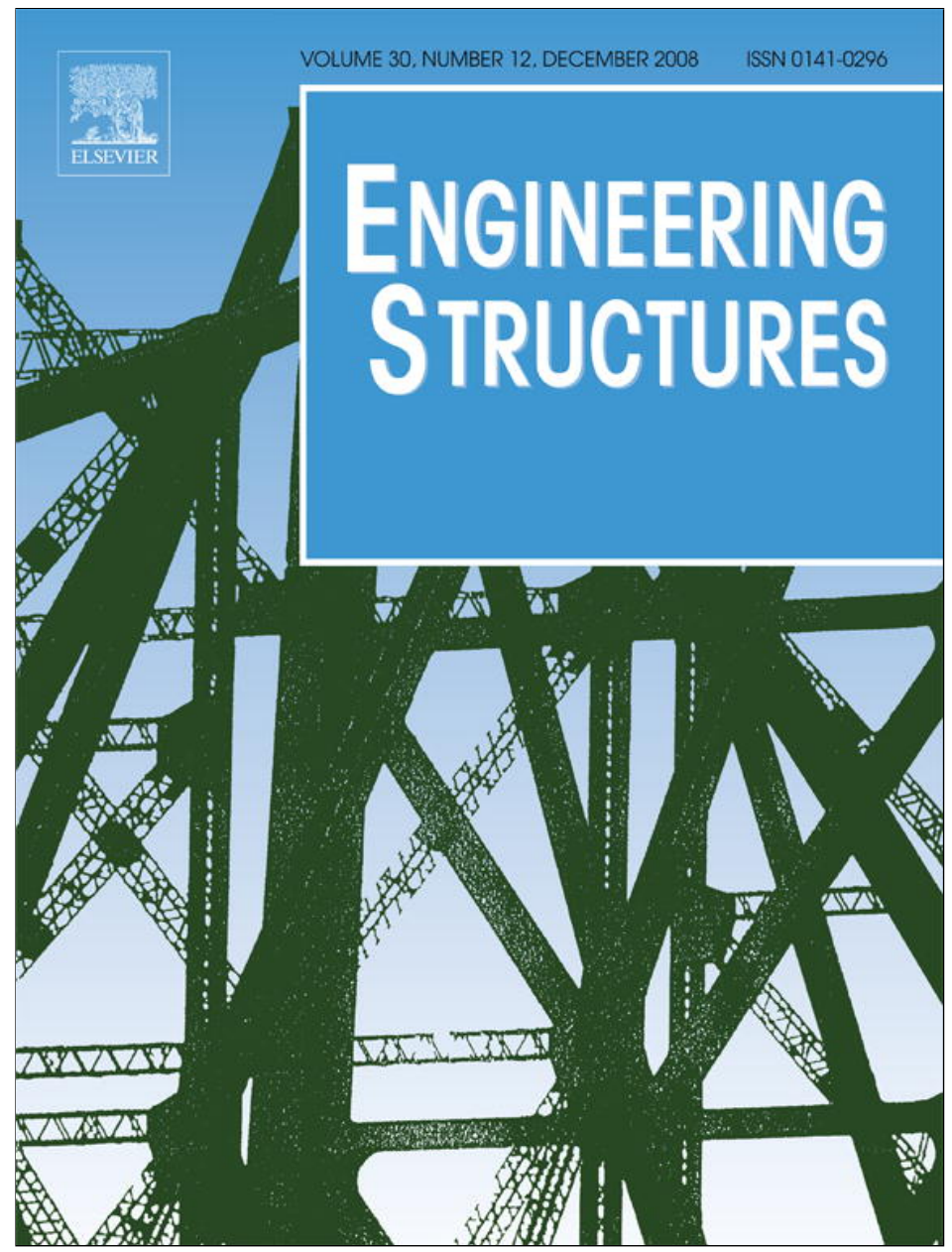

This article appeared in a journal published by Elsevier. The attached copy is furnished to the author for internal non-commercial research and education use, including for instruction at the authors institution and sharing with colleagues.

Other uses, including reproduction and distribution, or selling or licensing copies, or posting to personal, institutional or third party websites are prohibited.

In most cases authors are permitted to post their version of the article (e.g. in Word or Tex form) to their personal website or institutional repository. Authors requiring further information regarding Elsevier's archiving and manuscript policies are encouraged to visit:

http://www.elsevier.com/copyright 


\title{
Two-scale approach for the nonlinear dynamic analysis of RC structures with local non-prismatic parts
}

\author{
P. Mata, A.H. Barbat, S. Oller* \\ Departament de Resistència de Materials i Estructures a l'Enginyeria (RMEE), Universitat Politècnica de Catalunya (UPC), Jordi Girona 1-3, Mòdul C1. Campus Nord. 08034 \\ Barcelona, Spain
}

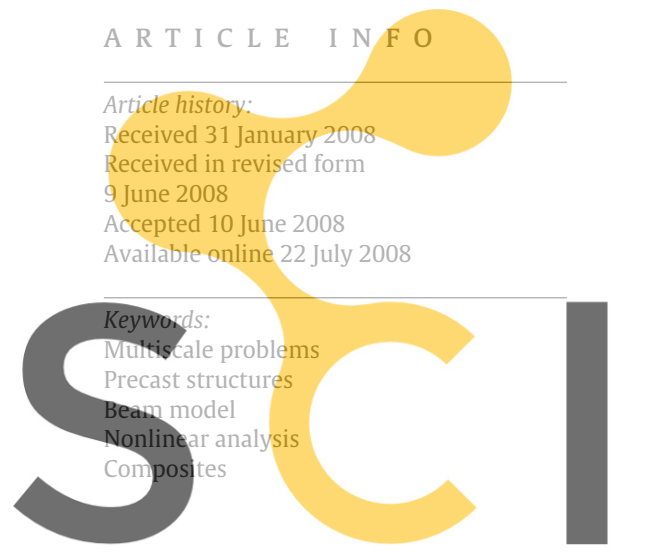

\begin{abstract}
A B S T R A C T
There is general agreement in the fact that fully three-dimensional (3D) numerical techniques provide the most precise tools for simulating the behavior of RC buildings even when their computational costs for real structures became them unpractical. Moreover, one-dimensional formulations (1D) are rather limited for predicting the mechanical behavior of framed structures which present local weakness that can determine their global responses, such as it is the case of poor detailed joints of RC buildings in seismic zones or precast concrete structures. An alternative approach, combining both simplicity

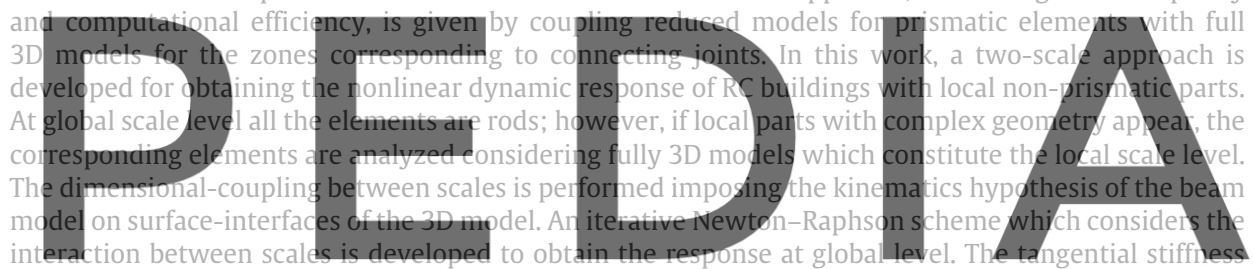
of the local models are obtained numerically. Computationally, the problem is managed by means of a master-slave approach, where the global scale problem.acts as the master and the local models are tangential tensors. The process stops when global convergence is achieved. From the computational point of view, the developed method is implemented in a parallelized scheme, where the master and slave problems are solved independently by different programs thus minimizing the intervention on existing codes specific for beams and solids. Finally, numerical examples are included.
\end{abstract}

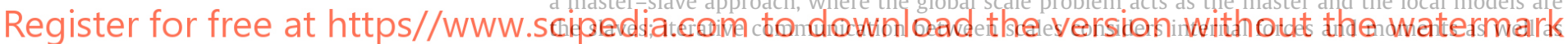

(C) 2008 Elsevier Ltd. All rights reserved.

\section{Introduction}

Fully 3D numerical techniques provide the most precise tools for simulating the nonlinear behavior of RC buildings, although the computing time required makes their applications unpractical [61]. Considering that a great part of the elements in buildings are prismatic, reduced (one-dimensional) formulations appear as a solution combining both numerical precision and reasonable computational costs [35,36]. Experimental evidence shows that inelasticity in beam elements can be formulated in terms of crosssectional quantities [5] and, therefore, the beam's behavior can be described by means of concentrated models, sometimes called plastic hinge models, which localizes all the inelastic behavior at the ends of the beam by means of ad-hoc force-displacement or moment-curvature relationships $[13,61]$. It is important to note

\footnotetext{
* Corresponding author. Tel.: +34 934016473; fax: +34 934011048.

E-mail address: sergio.oller@upc.edu (S. Oller).
}

that, in this case, the nonlinear constitutive laws are valid only for specific shapes of the cross sections and that frequently some components of the reduced forces and/or moments are treated elastically $[12,20,40,46]$. A further refinement in the analysis of the mechanical response of beam structures is obtained considering inhomogeneous distributions of materials on arbitrarily shaped cross sections [12]. In this case, the usual procedure consists of obtaining the constitutive relationship at cross-sectional level by integrating on a selected number of points corresponding to fibers directed along the beam's axis [53,62]. Thus, the mechanical behavior of beams with complex combinations of materials can be simulated $[3,10]$. Fiber models fall into the category known as distributed beam models [21] due to the fact that inelasticity can spread along the beam element axis [49]. In most cases, both types of models, the concentrated and the distributed ones, have been formulated under the hypothesis of infinitesimal deformation.

Geometric nonlinearity has mainly been treated by means of two different approaches: (i) The co-rotated or inexact formulations, which considers arbitrarily large displacements and 
rotations but the assumption of small strains as it can be reviewed in reference [11]. (ii) The geometrically exact formulations are obtained from the fully 3D theory of the continuum by means of reducing the number of dimensions using appropriated kinematic hypothesis [22]. One of the most successful geometrically exact formulation for beams is that due to Simo [56] and Simo and VuQuoc $[57,58]$ which extends to the three-dimensional case the formulation originally presented by Reissner $[50,51]$ and allows to consider finite extension, shearing, flexure and torsion, but with the restriction of maintaining the planarity of the cross sections. Additionally, other works based on alternative kinematic hypothesis allow considering the warping of the cross section [17,59]. In spite of the great capacity of the mentioned formulations, works considering both inelasticity and a geometrically exact kinematics are rather scarce. Some recent works can be reviewed, for example, in [7] a new (mesh independent) element for the full nonlinear analysis of RC plane frames based on the interpolation of the strains is presented. In [24] (see also references therein) Izzuddin and Lloyd Smith present a computationally efficient method for the analysis of RC structures based on adaptive analysis concepts which consider coupled geometric and constitutive sources of nonlinearity.

Most of the geometrically exact nonlinear models are limited to the elastic case $[56,57]$ and the inelastic behavior has been mainly restricted to plasticity $[17,52,59]$. Recently, Mata et al. $[35,36]$ have extended the geometrically exact formulation for beams of Reissner, Simo and others [23,25,27] including an arbitrary distribution of composite materials with inelastic

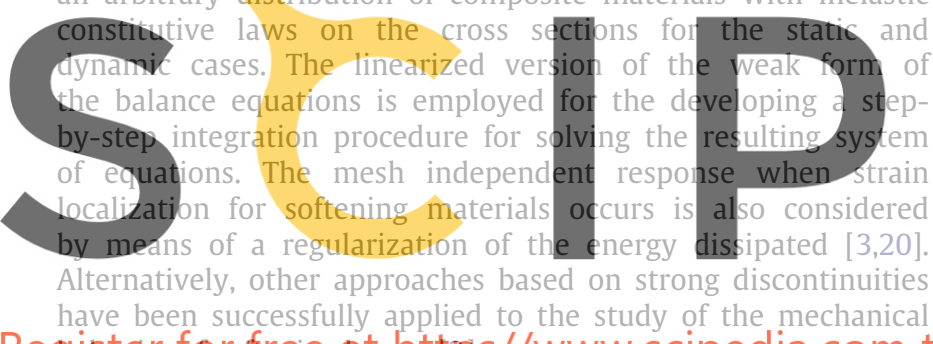

Registeer foroffeeeg atahttpis//www.scipedia.com to As it has been explained, although a great amount of work has been devoted to the development of refined nonlinear models for beam structures, ail of them are subjected to the foliowing limitations: (i) beam structures present either fully monolithic connections among elements or some of their degree of freedoms are released [26] (e.g. pinned connections). Therefore, structural failures due to damage inside of the nodes, such as those occurred in poorly designed RC buildings [6] or in the so called semi-rigid connections of steel or composite structures [1], are often not properly considered. (ii) The shape and the mechanical properties of the cross sections are considered constants in an element (or they have smooth variations) and, therefore, the beam models show serious difficulties in reproducing structural behaviors dominated by local changes in the geometry such as those observed in the connecting joints of precast concrete structures $[47,48]$.

Several attempts have been made for modeling the effects of local irregularities or local complex stress concentrations in framed structures. Probably, the most common approach consists of employing plastic hinge models with moment-curvature relations reflecting the mechanical characteristics of the connecting joints [29], although this approach has the previously described limitations. Other authors have focused their efforts towards developing specific solid-to-beam transition elements e.g. [64]. More realistic numerical studies of beam-column connections involve fully 3D finite element (FE) models as it is shown by Fu et al. in [14]; however, in their work the purpose still is obtaining simpler moment/curvature relationship for the connecting joints.

An alternative approach, combining precision, generality and computational efficiency consists of coupling reduced $1 \mathrm{D}$ and full
3D numerical models describing different parts of the structure. In this case, most of the elements are prismatic rods while the local non-prismatic parts or zones corresponding to the connecting joints receive a more detailed description. The connection between models of different dimensions is done through interface-surfaces. Research in this direction has been carried out by McCune et al. [38] where a scheme for establishing displacement compatibility and stress equilibrium at the interface is developed. The results are finally implemented as multi-point constraint equations relating the displacement field of the beam's node (which includes rotations) with the corresponding of the nodes of the solid at the interface. A similar approach is followed by Shim et al. [54,55] and an application to earthquake engineering can be reviewed in [18]. The kinematic restrictions for a consistent beam-to-shell transition element in finite deformation is presented in [63]. Garusi and Tralli [15] develop hypostatic transition solid-to-beam and plate-to-beam elements following a stress assumed method. In [19], the dimensional-coupling is obtained applying the Lagrange multiplier's method. In all the cases, the numerical examples are limited to the linear elastic case. In [16] a displacement based super-element is used for studying the propagation of waves in structures with singularities.

In this work, a two-scale approach (global and local) is developed in order to study the nonlinear response of RC framed buildings. At global scale level, all the elements of the FE model are rods in finite deformation; however, if (locally) geometric

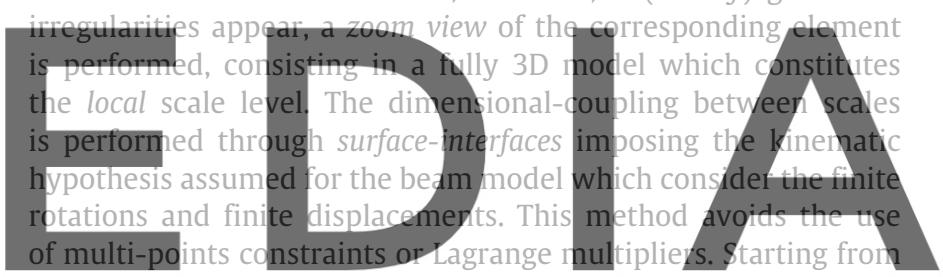

the full 3D stress state existing in the local model, cross-sectional forces and moments, required at global level, are recovered by

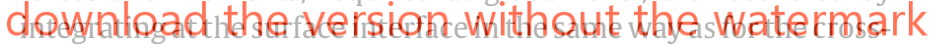
sectional analysis of beams [35]. An iterative Newton-Raphson scheme based on the displacement method, which considers the interaction between scales is developed to obtain the response at the global level even in the nonlinear dynamic range. Force and displacement equilibrium is checked at both, local and global levels, ensuring that compatible configurations are reached for the whole problem. The tangential stiffness of the local model is obtained numerically applying small perturbations on the current configuration and obtaining the corresponding reaction forces reduced to the degree of freedom of the global level.

From the point of view of the implementation in a numerical code, the problem is managed by means of a master-slave approach, where the global scale problem acts as the master, sending a trial displacement field to the local scale models (slaves) and then receives the corresponding internal forces, moments and tangential tensors estimated by means of integration on the surface interfaces. The iterative process is finished when the global convergence is achieved. Computationally, the proposed approach is well suited to be implemented in a parallelized algorithm, where the master and slave problems are solved independently by different programs. The communication between processes (and processors) is carried out by means of an appropriated library of communication. In this way, minimal intervention on existing codes (specific for beams and solids) allow obtaining the response of the whole structure in the nonlinear static and dynamic analysis. Finally, numerical examples are included showing the capabilities of the proposed formulation. 


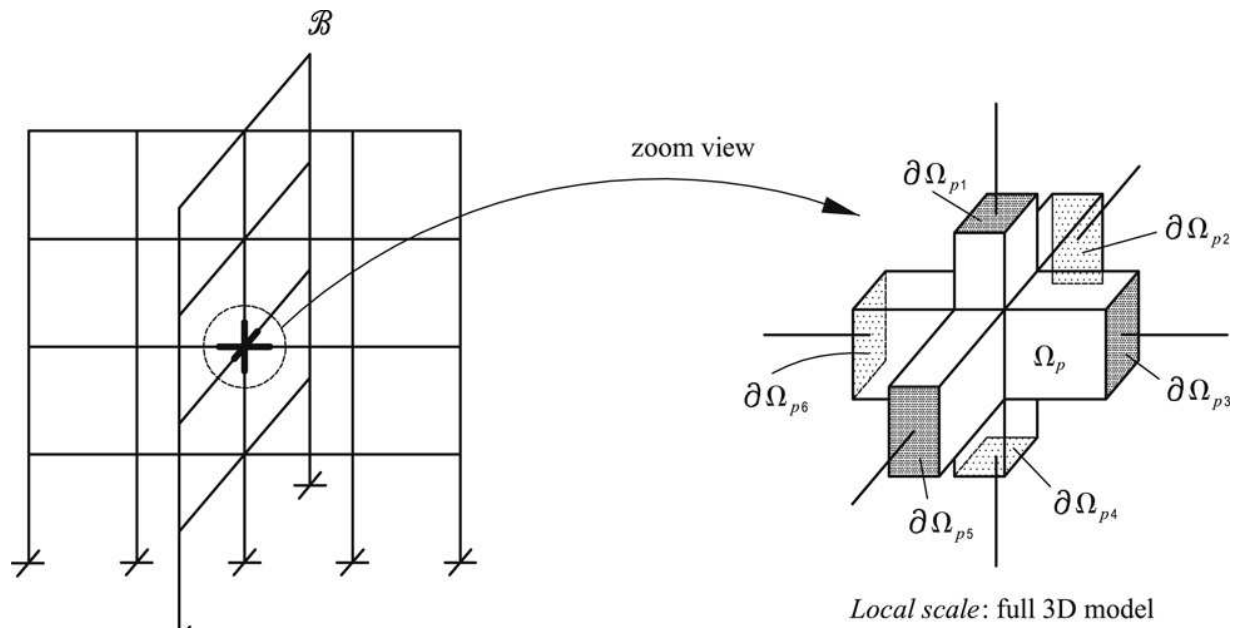

Global scale: beam model

\section{Description of the proposed model}

The whole body of the framed structure can be seen as divided in two different sets: (i) By one hand, $\mathcal{B}$ corresponds to the part of the body ${ }^{1}$ which is susceptible to be described by means of an of reduced $1 \mathrm{D}$ models whice by means of monolithic join $\left.\sum_{p} \Omega_{p s}\right\}$ is the set of $p=$ present local non-prismatic par $\mathcal{B}$ constitutes the globd vantages of the dimensiona to account, including geometr.

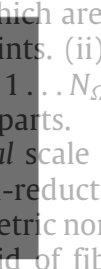
(ii) By t
$N_{\Omega}$ part
cale of the
duction o
c nonlinea

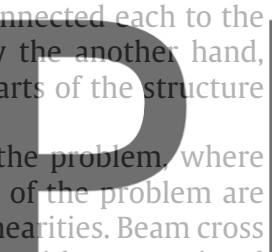
fibers with appropriated constitutive laws. The geometry and the distribution of materials

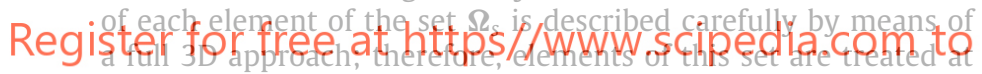
a more reduced scale, the local scale (in terms of the degree of detailing considered). The connection between $\mathcal{B}$ and one part $\boldsymbol{\Omega}_{p s}$ is obtained through the set $\partial \boldsymbol{\Omega}_{p s}:=\left\{\bigcup_{k} \partial \boldsymbol{\Omega}_{k p s}\right\}$, where $k=$ $1 \ldots N_{p \Omega}$ is an index which runs over the number of interfacesurfaces, $\partial \boldsymbol{\Omega}_{k p s} \in \mathbb{R}^{2}$. Each element of $\partial \boldsymbol{\Omega}_{p s}$ has one-to-one correspondence with the end cross section of a beam element connecting to $\boldsymbol{\Omega}_{p s}$ (See Fig. 1).

At global scale level, the so called dimensional-coupling [38,39] is obtained enforcing at local level, i.e. on each $\partial \boldsymbol{\Omega}_{k p s}$ of a given $\boldsymbol{\Omega}_{p s}$, the fulfillment of the kinematic hypothesis assumed for the $1 \mathrm{D}$ model. In the present work, planarity of the cross section is assumed during the motion and, therefore, the surface-interfaces of the local model displace and rotate as a rigid body and, in this sense, warping or cross-sectional distortion is not included. Reduced forces and moments from the local models are obtained though integration of the reaction forces in the interfaces in an analogous manner as for the cross- sectional analysis of beams [35]. The local model constitutes a zoom view made on a part of the structure, $\boldsymbol{\Omega}_{p s}$, which is treated at global level as an additional 1D element. Fig. 2 shows an illustrative example: a structure consisting of a 1D model and a 2D model deforms due to the motion $\phi(t)$ where $t$ is the time. It is possible to see that the cross section of the end of the beam corresponds to the surface interface of the $3 \mathrm{D}$ model, which moves as a $2 \mathrm{D}$ rigid body.

\footnotetext{
${ }^{1}$ By simplicity, in this work a part of a body is identified with all the material points which occupy a region on the 3D space and can be conveniently described using a coordinate system. For a more elaborated definition see [33].
}

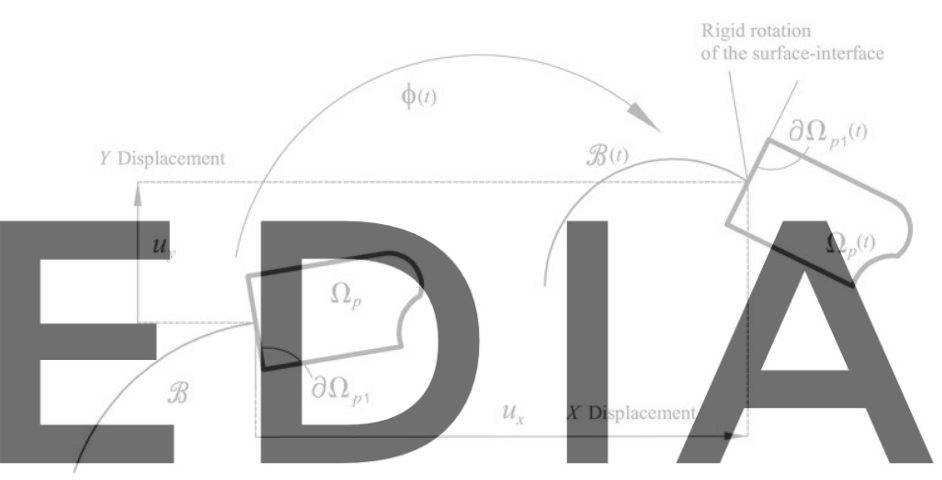

Fig. 2. Kinematics hypothesis of the 1D model imposed on the surface-interface download the version without the watermark

In other words, given a motion of the structure, the global scale only treats with dimensionally-reduced quantities as usual in beam's theories but the required quantities originating from elements of the set $\boldsymbol{\Omega}_{s}$ are obtained solving a 3D problem subjected to the beam kinematics's assumptions as boundary conditions on the displacements of $\partial \boldsymbol{\Omega}_{p s}$. In the following sections, a presentation of both, the local and the global models, as well as of the stepby-step numerical algorithm for solving the two-scale problem is given.

It is worth to note some advantages of the proposed approach:

(I) It avoids the construction of multi-point constraints relating the displacement (and rotation) fields at both scales as described, for example, in [38]. In general, the design of such type of constraints requires a rather significant number of hypothesis about the local scale stress field which are valid mainly in the elastic range and for simple (non-composite) materials. An extension of such an approach to the full nonlinear geometric and inelastic case is not obvious.

(II) Lagrange multipliers (see e.g. [19]) are a recognized method to enforce a large set of different types of kinematic conditions; however, in contrast with the present approach, the resulting global problem has an increased number of variables to be determined.

Remark 1. The proposed formulation can be seen as an ad-hoc numerical homogenization by means of an appropriated micro description of the domain corresponding to the local scale using 

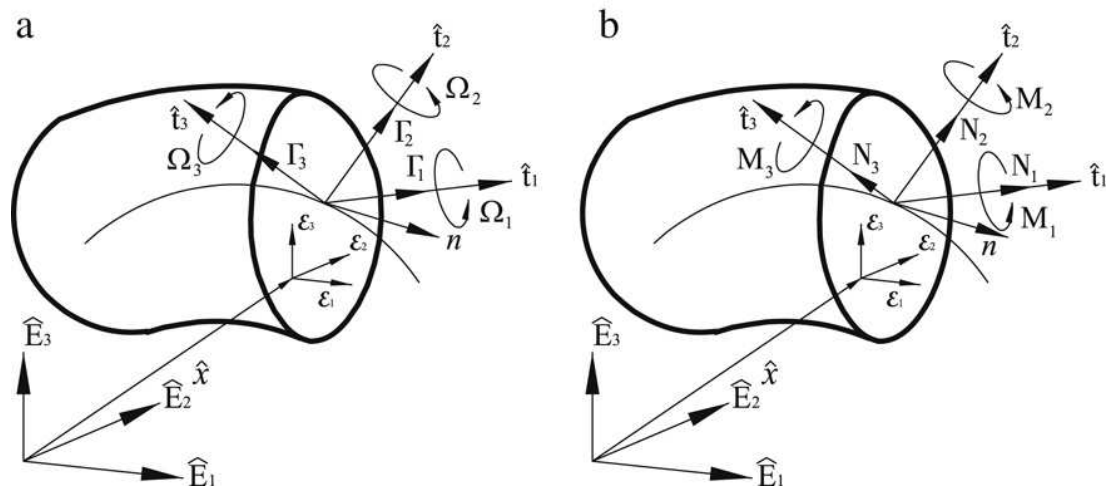

Fig. 3. Reduced cross-sectional quantities. (a): Strains. (b): Stress resultants.

the FEM. A similar approach has been used for the treatment of structures made of composite materials [45] in the context of $2 \mathrm{D}$ continuum problems.

\section{Global scale model: Beams}

The Reissner-Simo geometrically exact formulation for beams $[50,56,57]$ has been expanded by the authors for considering an inhomogeneous distribution of (inelastic) composite materials on the cross sections $[35,36]$. The FE implementation of this formulation is used as the global scale problem. In following some

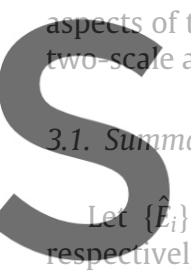
e approach, are reviewed.

mary of the theory

and $\left\{\hat{e}_{i}\right\}$ be the fixed
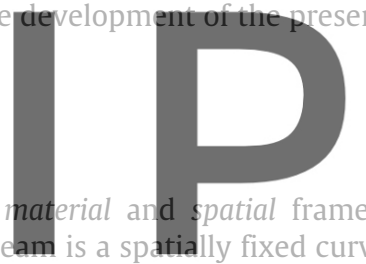

patial frames

$P_{0}(S) \in \mathbb{R}^{3}$, with $S \in[0, L]$ being the arch-length coordinate 0 in each $S$ there is rigidly attached an orthogonal local frame $\hat{t}_{0 i}(S)=$

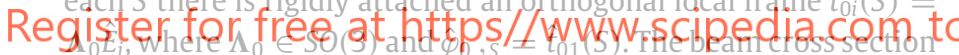
$\mathcal{A}(S) \in \mathbb{R}^{2}$ is defined considering the local coordinate system $\xi_{\beta}$ directed along $\left\{\hat{t}_{0 \beta}\right\}$

The deformation moves $\hat{\varphi}_{0}(S)$ to $\hat{\varphi}(S, t)$ at time $t$ adding a translational displacement and $\boldsymbol{\Lambda}_{0}(S)$ is rotated to $\boldsymbol{\Lambda}(S, t)=$ $\boldsymbol{\Lambda}_{n} \boldsymbol{\Lambda}_{0} \in S O$ (3) by means of the incremental rotation tensor $\boldsymbol{\Lambda}_{n}(S, t)$. In general, $\hat{t}_{1} \neq \hat{\varphi}{ }_{, S}$ because of the shearing [22]. The position vector of any material point with coordinates $\left(S, \xi_{\beta}\right)$ on the reference beam configuration, $\hat{x}_{0}$, and on the current beam configuration, $\hat{x}$, are given by

$\hat{x}_{0}=\hat{\varphi}_{0}(S)+\boldsymbol{\Lambda}_{0}(S) \xi_{\beta} \hat{E}_{\beta}, \quad \hat{x}=\hat{\varphi}(S, t)+\boldsymbol{\Lambda}(S, t) \xi_{\beta} \hat{E}_{\beta}$,

respectively. Then the displacement field on each material point on a cross section $\mathcal{A}(S)$, at time $t$, is obtained as

$\hat{u}\left(S, \xi_{\beta}, t\right)=\hat{x}-\hat{x}_{0}$.

Eq. (1) implies that the current configuration is determined by $(\hat{\varphi}, \boldsymbol{\Lambda}) \in \mathbb{R}^{3} \times S O(3)$ [57] and a kinematically admissible variation field ${ }^{3}$ is $(\delta \hat{\varphi}, \delta \hat{\theta}) \in \mathbb{R}^{3} \times T_{\Lambda}^{\text {spa }}$.

The deformation gradients of the curved reference beam and of the current beam are denoted by $\boldsymbol{F}_{0}$ and $\boldsymbol{F}$, respectively; (see [35]). Then, $\boldsymbol{F}_{n}:=\boldsymbol{F F}_{0}^{-1}$ relates differential arch-length elements of

\footnotetext{
2 Latin indices run over 1 to 3 and Greek indices over 1 to 2 . The symbol $(\bullet),{ }_{x}$ is used to denote partial differentiation of $(\bullet)$ with respect to the variable $x$.

${ }^{3}$ The symbol $T_{\Lambda}^{\text {spa }}$ is the spatial linear vector space of rotations on $\mathrm{SO}(3)$ with base point $\boldsymbol{\Lambda}$. For more details consult [32].
}

the curved reference configuration with the corresponding in the current placement and it is possible to construct the strain tensor $\varepsilon_{n}=F_{n}-\Lambda_{n}$. The associated spatial strain vector acting on a material point on the current beam cross section is

\section{$\hat{\varepsilon}_{n}=g_{0}^{-1}\left(\hat{\gamma}_{n}+\hat{\omega}_{n} \times \xi_{\beta} \hat{t}_{\beta}\right)$}

where $g_{0}$ is the determinant of $\boldsymbol{F}_{0}, \hat{\gamma}_{n}=\hat{\varphi},{ }_{s}-\hat{t}_{1}$ measures elongation and shearing $[23,56]$ and $\hat{\omega}_{n}$ is the current curvature vector relative to the curved reference beam (the axial vector of $\left.\widetilde{\omega}_{n} \equiv \Lambda_{n},{ }_{S} \Lambda_{n}^{T}\right)$. The material version of $\hat{\varepsilon}_{n}$ is given by

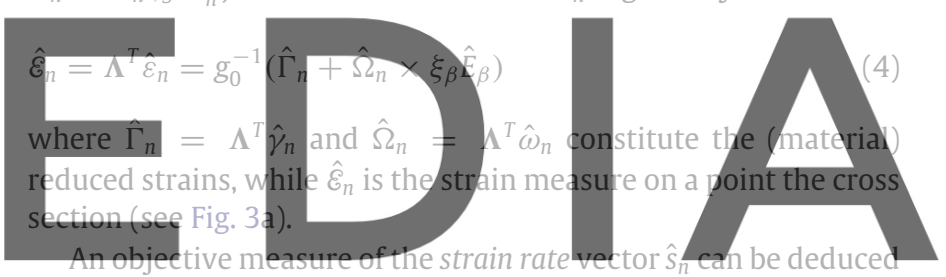
using the Lie derivative operator [35] as

\section{download the version without the watermark}

where $\widetilde{v}_{n}=\dot{\Lambda}_{n} \Lambda_{i n}^{T}$ is the spatial angular velocity tensor referred to the curved reference beam (see [36]). The corresponding material form is $\hat{s}_{n}=\Lambda^{\mathrm{T}} \hat{s}_{n}$.

Lets $\hat{P}_{1}$ be the First Piola Kirchhoff (FPK) stress vector (on a point of the cross section) referred to the curved reference beam, which is energetically conjugated to $\hat{\varepsilon}_{n}$. Integrating over the cross section, it is possible to obtain the stress resultant $\hat{n}$ and the stress couple $\hat{m}$ according to

$\hat{n}(S)=\int_{\mathcal{A}} \hat{P}_{1} \mathrm{~d} \mathcal{A} ; \quad \hat{m}(S)=\int_{\mathcal{A}}(\hat{x}-\hat{\varphi}) \times \hat{P}_{1} \mathrm{~d} \mathcal{A}$

with material forms given by $\hat{P}_{1}^{\mathrm{m}}=\boldsymbol{\Lambda}^{\mathrm{T}} \hat{P}_{1}, \hat{\mathrm{N}}=\boldsymbol{\Lambda}^{\mathrm{T}} \hat{n}$ and $\hat{\mathrm{M}}=\boldsymbol{\Lambda}^{T} \hat{m}$, respectively (see Fig. $3 \mathrm{~b}$ ).

The reduced balance equations referred to the curved reference beam, can be written as [27]

$\hat{n},_{S}+\hat{n}_{p}=A_{\rho 0} \ddot{\hat{\varphi}}, \quad \hat{m},_{S}+\hat{\varphi},_{S} \times \hat{n}+\hat{m}_{p}=\boldsymbol{l}_{\rho 0} \hat{\alpha}_{n}+\widetilde{\boldsymbol{v}}_{n} \boldsymbol{l}_{\rho 0} \hat{v}_{n}(7)$

where $\hat{n}_{p}$ and $\hat{m}_{p}$ are the external (applied) force and moment per unit of reference length, respectively; $\hat{\alpha}_{n}$ is the angular acceleration vector, $A_{\rho 0}$ and $\boldsymbol{l}_{\rho 0}$ are the mass density and the second mass moment density per unit of length of the curved reference beam, respectively $[27,57]$.

Considering an admissible variation $\hat{h}=(\delta \hat{\varphi}, \delta \hat{\theta})$ [57], taking the dot product with Eq. (7) after some manipulations, one may 
obtain the functional $\mathrm{G}_{G}(\hat{\varphi}, \boldsymbol{\Lambda}, \hat{h})$ corresponding to the virtual work principle:

$$
\begin{aligned}
\mathrm{G}_{G}= & \underbrace{\int_{L}\left[\delta \stackrel{\nabla}{\gamma} \hat{n}+\delta \stackrel{\nabla}{\omega}_{n} \cdot \hat{m}\right] \mathrm{d} S}_{\mathrm{G}_{\mathrm{Gint}}(\hat{\varphi}, \boldsymbol{\Lambda}, h)} \\
& +\underbrace{\int_{L}\left[\delta \hat{\varphi} \cdot A_{\rho_{0}} \ddot{\hat{\varphi}}+\delta \hat{\theta} \cdot\left(\boldsymbol{l}_{\rho_{0}} \hat{\alpha}_{n}+\widetilde{\boldsymbol{v}}_{n} \boldsymbol{l}_{\rho_{0}} \hat{v}_{n}\right)\right] \mathrm{d} S}_{\mathrm{G}_{\mathrm{Gine}}(\hat{\varphi}, \boldsymbol{\Lambda}, h)} \\
& -\underbrace{\int_{L}\left[\delta \hat{\varphi} \cdot \hat{n}_{p}+\delta \hat{\theta} \cdot \hat{m}_{p}\right] \mathrm{d} S}_{\mathrm{G}_{\mathrm{Gext}}(\hat{\varphi}, \boldsymbol{\Lambda}, h)}-\left[\left.(\delta \hat{\varphi} \cdot \hat{n})\right|_{0} ^{L}+\left.(\delta \hat{\theta} \cdot \hat{m})\right|_{0} ^{L}\right] \\
= & 0
\end{aligned}
$$

where $\delta \stackrel{\nabla}{\gamma}$ and $\delta \stackrel{\nabla}{\omega}_{n}$ are the co-rotated variations of $\hat{\gamma}$ and $\hat{\omega}_{n}$, respectively. The functional $G_{G}$ is the sum of the internal, inertial and external contributions, $G_{G i n t}, G_{G i n e}$ and $G_{G e x t}$, respectively.

\subsection{Constitutive laws}

Points on the cross sections are assumed to have associated a composite material as explained in [44]. The composite is formed by several simple constituents which can have associated any of the following constitutive models: linear elasticity, rate independent plasticity and damage or visco damage. All these models have been developed considering a suitable form compatible with the kinenyatics described in Eq. (1) and the details can be consylted in [35,36]. For the purpose of explaini mulation it is enough to say that each lowing way:
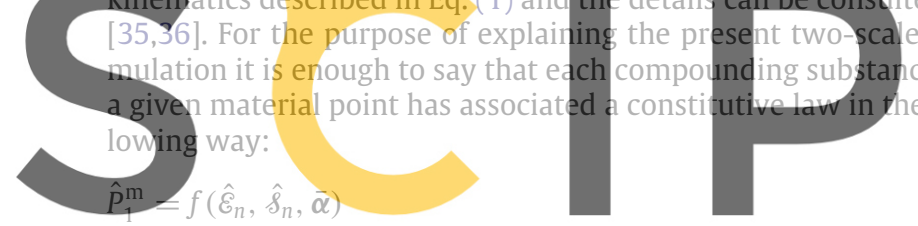

(9)

where $\hat{P}_{1}^{\mathrm{m}}$ and $\hat{\mathscr{E}}_{n}$ are the material form of the FPK stress and strain

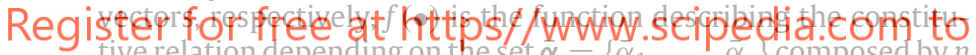
tive relation depending on the set $\alpha=\left\{\alpha_{1}, \ldots, \alpha_{n}\right\}$ composed by $n$ internal variables. Additionally, a tangent consecutive relation between linearized increments of strain and stress exist and is expressed as

$\delta \hat{P}_{1}^{\mathrm{m}}=\boldsymbol{e}^{\mathrm{mt}} \delta \hat{\varepsilon}_{n}$.

The mixing rule for composites [9] is used to obtain the resulting stress and tangential tensor at material point level according to

$\hat{P}_{1}^{\mathrm{m}}=\sum_{p=1}^{N_{c}} k_{p}\left(\hat{P}_{1}^{\mathrm{m}}\right)_{p}, \quad \overline{\boldsymbol{e}}^{\mathrm{mt}}=\sum_{p=1}^{N_{c}} k_{p}\left(\boldsymbol{e}^{\mathrm{mt}}\right)_{p}$

where $\left(\hat{P}_{1}^{\mathrm{m}}\right)_{p}$ and $\left(\boldsymbol{e}^{\mathrm{mt}}\right)_{i}$ are the stress vector and tangential tensor for the $p=1 \ldots N_{c}$ compounding substances which participates according to their volumetric fraction $k_{p}$ [9]. On the other hand, a mesh independent response of the structure is obtained regularizing the dissipated energy at constitutive level as explained in reference [35].

\subsection{Damage index}

The details of the damage index, $\check{D}$, used in this work can be consulted in references $[35,36]$. It constitutes a measure of the damage level of a material point obtained as the ratio of the existing stress level to its visco elastic counter part as follows:

$\sum_{i}\left|P_{1 i}^{\mathrm{mt}}\right|=(1-\check{D}) \sum_{i}\left|P_{1 i 0}^{\mathrm{mt}}\right|=\rightarrow \check{D}=1-\frac{\sum_{i}\left|P_{1 i}^{\mathrm{mt}}\right|}{\sum_{i}\left|P_{1 i 0}^{\mathrm{mt}}\right|}$ where $\left|P_{1 i}^{\mathrm{mt}}\right|$ and $\left|P_{1 i 0}^{\mathrm{mt}}\right|$ are the absolute values of the components of the existing (rate dependent) and visco elastic stress vectors, respectively. Initially, $\check{D}=0$, but when the entire fracture energy of the material is dissipated $\left|P_{1 i}^{\mathrm{mt}}\right| \rightarrow 0$ and $\check{D} \rightarrow 1$. Eq. (12) can be applied to consider a structural member, a part of the structure or the whole structure, by integrating the stresses over the corresponding volume of the structure.

\section{Local model: 3D connecting joint}

Once a local non-prismatic part has been detected on a given rod, a zoom view, corresponding to the local 3D scale, is opened. This section is devoted to explain the mechanical problem which has to be solved at local level. It is worth to recall that the dimensional-coupling between scales is obtained by imposing the plane section assumption for beams on the surface-interfaces in the 3D model (see Fig. 2).

\subsection{Equilibrium equations and boundary conditions}

Assuming a massless local model $\left(\rho_{0}=0\right)$, we obtain that points on the (plane) surface-interfaces are subjected to the same motion as the corresponding cross sections of the beams connected to them. At local level, the momentum balance equations of the

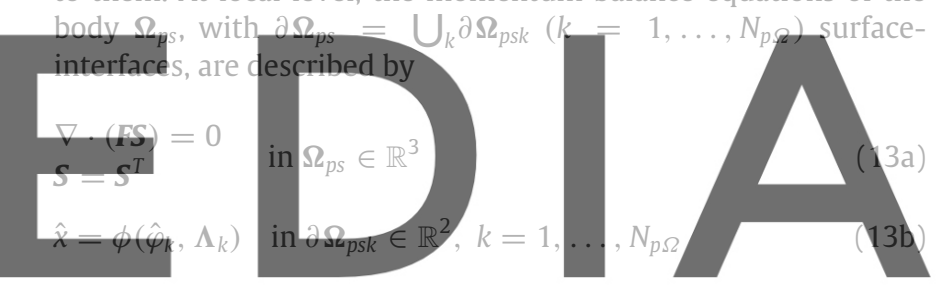

where $S$ is the second Piola-Kirchhoff (SPK) stress tensor, no external body forces are applied i $\hat{b}_{0}=0$ as well as prescribed

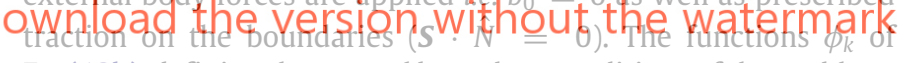
Eq. (13b), defining the natural boundary conditions of the problem, are prescribed in accordance with Eq. (1) and they are expressed ${ }^{4}$ as

$\hat{x}_{L}=\hat{\varphi}_{k}-\hat{\varphi}_{0 k}+\chi_{\beta}\left(\boldsymbol{\Lambda}_{k}-\boldsymbol{\Lambda}_{0 k}\right) \hat{E}_{\beta}=\hat{u}_{k} ; \quad \forall \hat{x}_{L} \in \partial \boldsymbol{\Omega}_{p s k}$

where $\hat{\varphi}_{k}, \hat{\varphi}_{0 k}, \boldsymbol{\Lambda}_{k}, \boldsymbol{\Lambda}_{0 k}$ are the current and reference displacement fields and rotation tensors associated to the beam connecting to the $k$ th surface-interface, $\hat{u}_{k}$ is the corresponding displacement, $\chi_{\beta}$ are the local (orthogonal) coordinates of the material point $\hat{x}_{L}$ lying on the surface-interface $\partial \boldsymbol{\Omega}_{p s k}$. In an analogous manner as for the finite strain beam theory $\chi_{\beta}$ are directed along a movable local axis $\hat{t}_{k(\beta)}=\Lambda_{k} \hat{E}_{\beta}$ (see Fig. 4).

Following standard arguments in continuum mechanics [33] we have that the weak statement of Eqs. (13a) and (13b) is given by

$G_{L}(\hat{x}, \delta \hat{x})=\int_{\boldsymbol{\Omega}_{p s}(t=0)} \boldsymbol{S}: \delta \boldsymbol{E} \mathrm{d} V=0$

where $\boldsymbol{E}$ is the Green-Lagrange (GL) strain tensor energetically conjugated to $\boldsymbol{S}$ and $t$ the time. It is worth to note that the strain and stress fields developed in the body $\boldsymbol{\Omega}_{p s}$ during the motion, are derived from the imposition of the displacement field of Eq. (13b).

\footnotetext{
${ }^{4}$ The subscript $L$ has been introduced to highlight that the respective quantity is referred to the local model.
} 


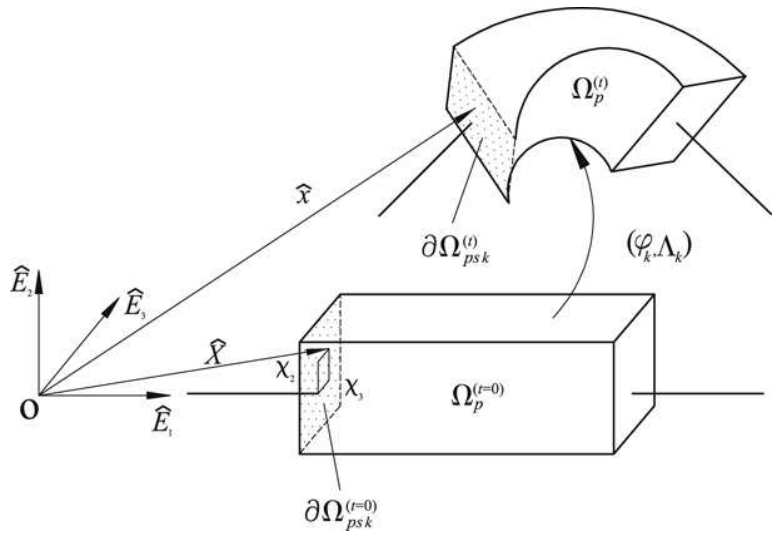

Fig. 4. Time evolution of the material points lying on the surface-interfaces.

\subsection{Constitutive equations}

Material points on $\Omega_{p s}$ are assumed to have associated a composite $[9,44]$ formed by several simple constituents with their own constitutive models. In this case, the GL tensor $\boldsymbol{E}$ is complete in the sense that all the 9 components can be determined from a given displacement field; therefore, rate dependent and independent constitutive models for plastic and degrading materials can be formulated based on a solid thermodynamic basis. Additionally, the mixing rule is used to treat the resulting composite [9]

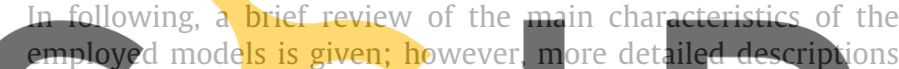
employed models is given; however can be consulted in previous works of Particularly, concrete is treated by nodel, where the degrading behav conducted by the evolution of a scalar over [0.1] (0 for the undamaged behavior and 1 for a complete ore detail
of a mathors
paramete d descriptions
$9,30,41,43,44]$.
tropic damage
erial point is degradation). The model is able to differentiate between tensile and compressive properties of the material. A detailed description 作 model of Ref. [31].

The longitudinal reinforcement can be seen as a material which has elastic plastic behavior directed along the line of the reinforcing bars. A plastic model for orthotropic materials is used to simulate the mechanical behavior of steel reinforcements [9]. Imposing the mechanical properties of the concrete in the direction perpendicular to the axis of the reinforcing bar and orienting the plastic flow in the direction of the reinforcement, it is possible to simulate the typical behavior of the steel bars.

From the point of view of the construction of a FE model of the local non-prismatic part, one proceed meshing the geometry as usual; then the material associated to elements located in places where steel reinforcements are present, is considered as a composite material according to the mixing rule $[43,44]$. The composite material is made of two phases: (i) A concrete phase described by means of a degrading material and (ii) a phase corresponding to the content of steel reinforcements (longitudinal and/or transversal). The mechanical properties of the steel phase are defined in a manner such that the directionality of the reinforcement is considered.

Fig. 5 shows the 3D joint of a typical RC structure. The view $A-A$ shows a typical FE discretization of one of its cross sections where three kinds of materials are used: (i) a purely damaging model for the elements of the unconfined zone. (ii) A coupled plastic damage model for the confined zone of the concrete. (iii) A composite material with three phases for the zone where longitudinal and transversal steel reinforcements appear altogether with the concrete. Each phase of the mixture contributes according to its volumetric fraction [34]. The proposed approach for the local scale model has the advantage of avoiding the usage of highly refined FE meshes.

Remark 2. A really large number of works has been devoted to the development of models for concrete including complex phenomena such as intrinsic anisotropy, creep and fracture among others (see e.g. [42]); however, the main contributions of the present work, which are focused on the treatment of local irregularities in buildings in finite deformation, are independent of the constitutive model used

\subsection{Cross-sectional and boundary surface analysis}

From the point of view of the numerical implementation, a beam cross section is meshed into a grid of quadrilaterals, each of them corresponding to a fiber oriented along the beam axis. An appropriated cross-sectional analysis for fiber beam models in finite deformation has been presented in references [35,36]. In those works, an integration of the constitutive equation of each simple material existing on each fiber level is followed by the application of the mixing rule and a posterior integration on the cross-sectional surface. The same procedure allows to determine the cross-sectional tangential stiffness.

In the case of the dimensional-coupling between scales, after a displacement field in the local scale problem fulfilling Eqs. (13a) and (13b) has been determined, it remains opened the question

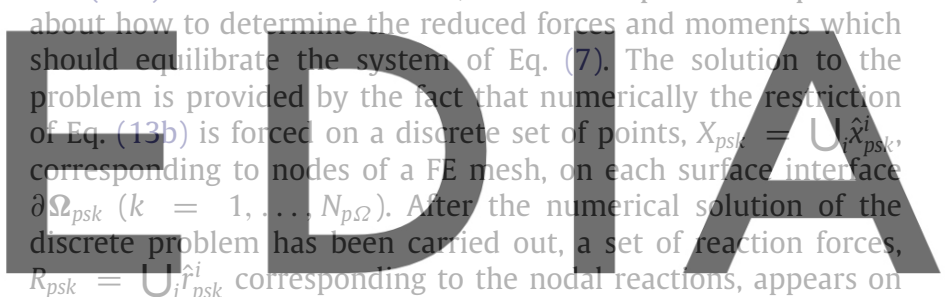
each of the $\partial \Omega_{p s k}$. The reduced forces and moments acting on the

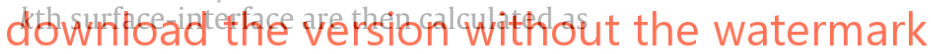
$\hat{N}_{G}=\sum \hat{r}_{p s k}^{i}, \quad \hat{M}_{G}=\sum \tilde{l}_{i} \hat{r}_{p s k}^{i}$

where $\hat{N}_{G}, \hat{M}_{G}$ are the reduced force and moment vector obtained from the full 3D problem of the local scale, $\widetilde{l}_{i}$ is the skew-symmetric tensor obtained from $\hat{l}_{i}=\left(\chi_{2}, \chi_{3}\right)_{i}$, the vector of coordinates of the $i$ th restricted point on the $k$ th surface-interface.

Remark 3. It is worth to note that in order to achieve equilibrium at global level, the reduced forces and moments obtained from the global and local scales have to self-equilibrate. Considering that the displacement and rotation fields are the same for both scales due to the kinematics assumption, one obtains that this condition enforces that the work done by both models is the same at the surface-interfaces

\section{Numerical procedure}

In this section a Newton type numerical solution procedure based on the linearized form of the weak form of Eq. (8) is developed [23,57]. The interaction between scales is explained as well as the method used to obtain the reduced tangential stiffness tensor of the local scale problem.

\subsection{Global scale: Consistent linearization for beams}

At global scale level each element behaves as a rod, therefore, the numerical solution for most of the elements is based 


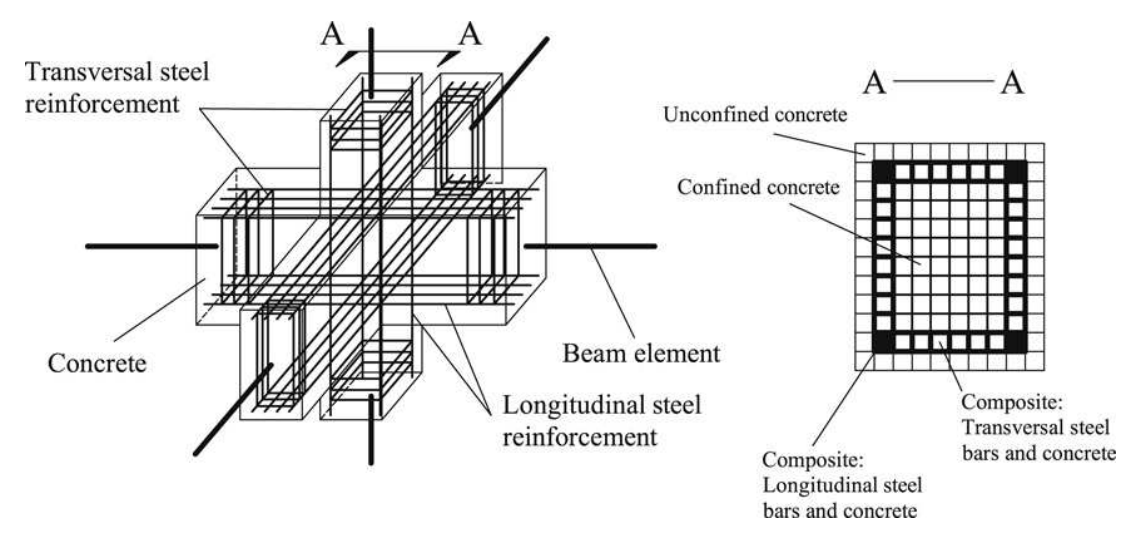

Fig. 5. FE model of the local scale including directional reinforcements.

linearizing the functional of Eq. (8) which can be written as

$\mathscr{L}\left[G_{G *}(\hat{h})\right]=G_{G *}(\hat{h})+D G_{G *}(\hat{h}) \cdot \hat{p}$

where $\mathscr{L}\left[\mathrm{G}_{G_{*}}(\hat{h})\right]$ is the linear part of $\mathrm{G}_{G}$ at the configuration defined by $\left(\hat{\varphi}_{*}, \Lambda_{*}\right)$ and $\hat{p} \equiv(\Delta \hat{\varphi}, \Delta \hat{\theta})$ is an admissible variation. The term $G_{G *}(\hat{h})$ supplies the unbalanced force and $D G_{G *}(\hat{h}) \cdot \hat{p}$ gives the tangential stiffness. ${ }^{5}$

In the general (inelastic) case, Eq. (17) can be rewritten as

$\mathscr{L}\left[\mathrm{G}_{\mathrm{G} *}\right]=\mathrm{G}_{\mathrm{G} *}+\left[\mathrm{K}_{M}+\mathrm{K}_{G}+\mathrm{K}_{V}+\mathrm{K}_{P}+\mathrm{K}_{\text {ine }}\right]_{*}$

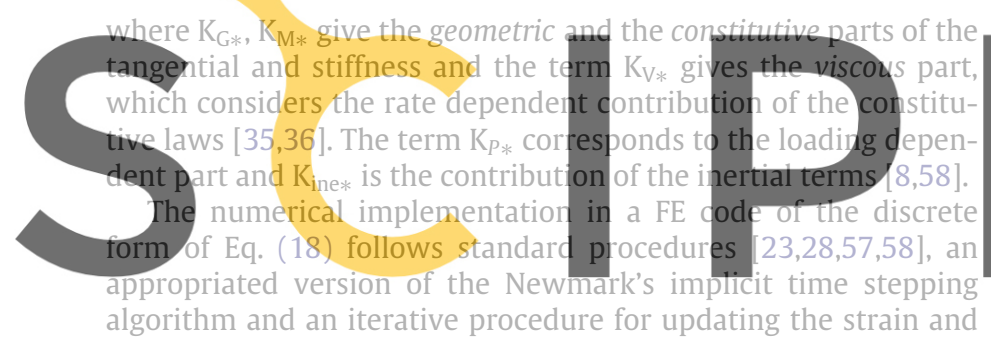

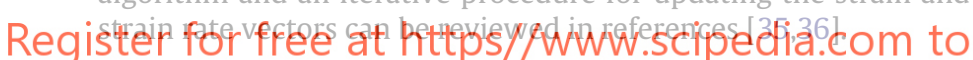

\subsection{Local scale: FE model and reduced tangential stiffness}

At global scale level, an iterative step-by-step Newton-Raphson scheme is used to solve the discrete version of the linearized form of the functional, $G_{L}$, given in Eq. (15), considering the boundary conditions of Eq. (13b). The usual FE procedure is applied as described, for example, in [4]; therefore, details are omitted here. After convergence is achieved, the reduced forces and moments corresponding to the surface-interfaces are estimated from the reaction forces as explained in Section 4.3.

The another ingredient required in the global scale is the reduced tangential tensor, i.e. the tangential stiffness obtained from the 3D model but reduced to the beam's degrees of freedom (DOFs) connected to the surface-interfaces. This tensor is obtained by means of applying the perturbation method [9] as follows:

(i) Supposing a local scale model used to describe the solid $\boldsymbol{\Omega}_{p s}$, with $\partial \boldsymbol{\Omega}_{p s}=\bigcup_{k} \partial \boldsymbol{\Omega}_{p s k}\left(k=1, \ldots, N_{p \Omega}\right)$ surfaceinterfaces, there are $\left(6 \times N_{p \Omega}\right)$ DOFs corresponding to the beams connected to the solid. ${ }^{6}$ After a displacement field fulfilling Eqs. (13a) and (13b) (in the nonlinear range) has been determined, a set of perturbations on each one of the $\left(6 \times N_{p \Omega}\right)$ DOFs is performed. In this case a perturbation in the $p$ th DOF

\footnotetext{
${ }^{5}$ Details can be consulted in [23,57].

6 They correspond to 3 displacements and 3 rotation on each surface-interface connected to the solid.
}

consist in imposing of a displacement field with a magnitude close to the numerical precision of the computer machine on the nodes of the corresponding surface-interface. ${ }^{7}$ Let denote one of such perturbations by $\delta U_{G}^{p}$.

(ii) For the new boundary condition corresponding to each $\delta U_{G}^{p}$, the problem defined by Eqs. (13a) and (13b) is solved and then the corresponding increments in the reaction forces of the restricted nodes of the body are calculated.

(iii) By using the formulas of Eq. (16), the increments of the reduced forces and moments are calculated. Let us denote

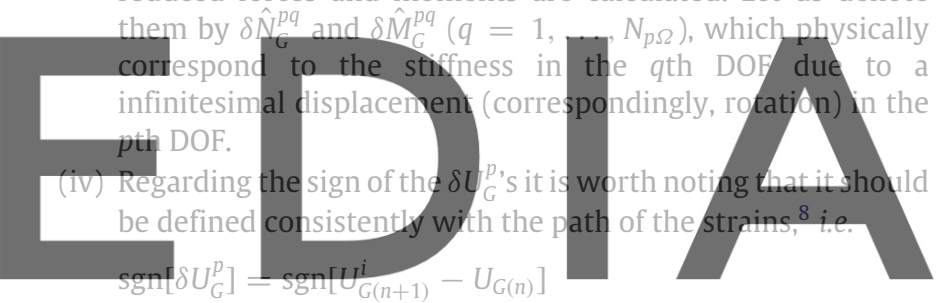

where $U_{G(1)+1)}^{i}$ denotes the global displacement (equivalently,

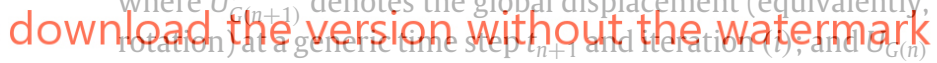
is its converged value at $t_{n}$. In this manner, the perturbation $\delta U_{G}^{p}$ inherit the tendency (loading or unloading) of the global scale deformation. If $\operatorname{sgn}\left[\delta U_{G}^{p}\right]<0$, unloading is induced in the $p$ th DOF.

Therefore, the numerical estimation tangential stiffness of the local scale model reduced to the global DOFs is given by

$[\mathscr{K}]_{L}^{\mathrm{t}}=\left[\begin{array}{ccc}\delta \hat{N}_{G}^{11} & \cdots & \delta \hat{N}_{G}^{1 q} \\ \vdots & \ddots & \vdots \\ \delta \hat{M}_{G}^{q 1} & \cdots & \delta \hat{N}_{G}^{q q}\end{array}\right]$.

Initially, the tangent stiffness of Eq. (19) corresponds to the elastic one, $[\mathscr{K}]_{L}^{0}$, and in this case, the applied perturbation can be small or large.

Remark 4. Note that under certain circumstances, step (iv) of the above list may yield to a situation where the body at local scale remains in the elastic range. However, after applying the perturbation, nonlinear incursions appear, affecting the values of the obtained stiffness matrix and the convergence of the method.

\footnotetext{
7 Perturbations on translational DOFs impose translational displacement fields on the nodes of the surface-interface and perturbations on rotational DOFs impose infinitesimal (additive) rigid body rotations.

$8 \operatorname{sgn}[\bullet]$ denotes the sign of $(\bullet)$.
} 


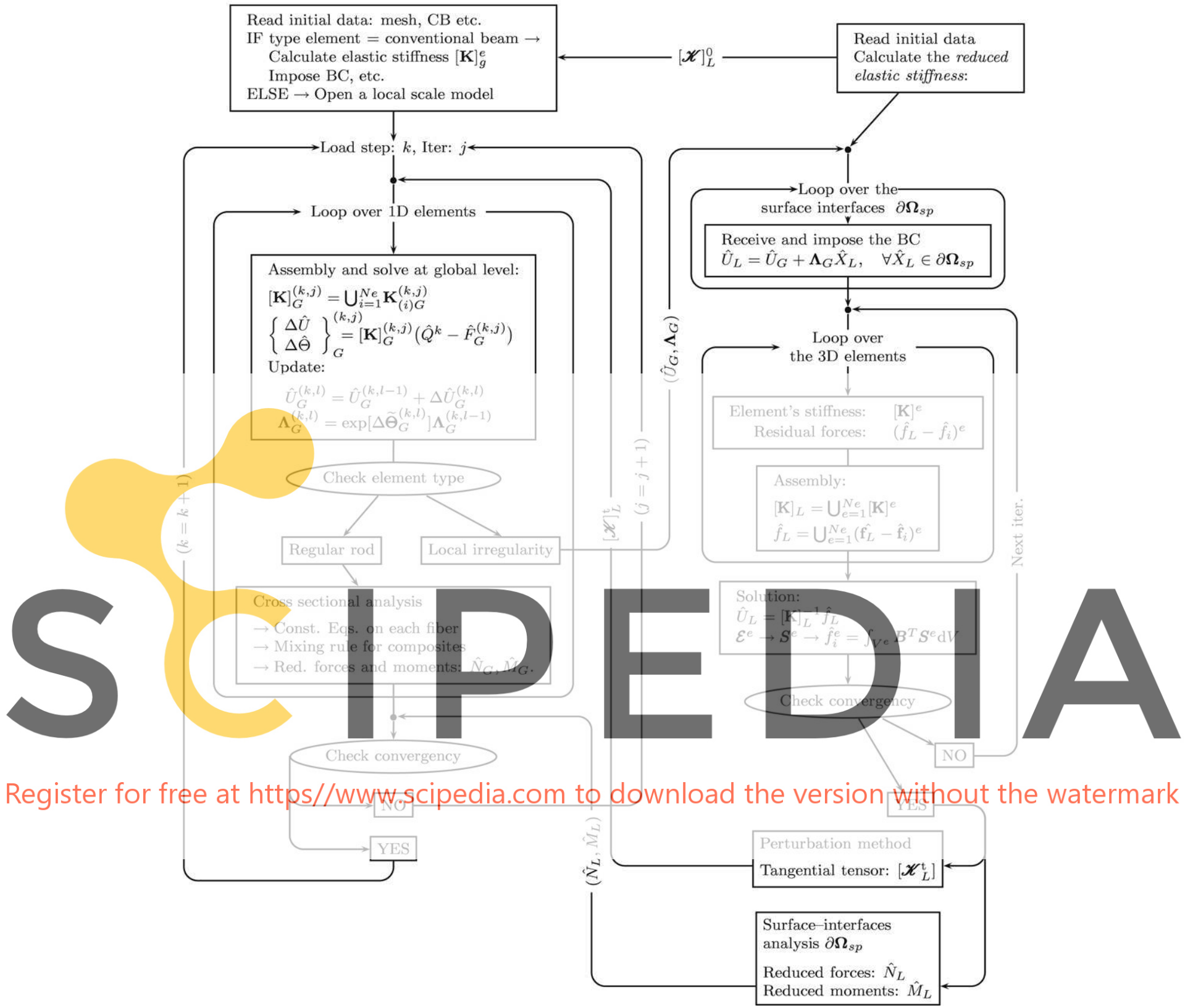

Fig. 6. Flow chart of the two-scale Newton-Raphson iterative scheme.

\subsection{Two-scale Newton-Raphson integration procedure}

As it has been explained before, the two scales are dimensionally-coupled through the reduced forces and moments and the tangential tensors. From the numerical point of view, at global scale level, once a loading step is applied, the corresponding displacement and rotation fields are obtained on the nodal DOFs. After that, the following sequence of steps is carried out:

(i) If the element corresponds to a classical rod, the strain fields are calculated on each fiber of the cross sections corresponding to an integration point and the reduced forces, moments and tangential matrices are determined as described in [35, 36 ]. If the element corresponds to a local non-prismatic part, the full 3D problem at local scale level is solved using an iterative Newton-Raphson scheme in the FEM and imposing the rod's kinematics assumptions on the surface interfaces. In the nonlinear range, several iterations can be needed at local scale level to obtain the converged field which provides the not necessarily convergent (in a global sense) reduced forces and moments at global scale level.

(ii) After convergence is achieved at local scale level the obtained reduced forces, moments and tangential tensor are sent to the global iterative scheme to the check convergence. If this is the case: (1) the local data base is updated and (2) the global algorithm proceed to apply a next load increment. If not, a new iteration is performed at global level.

The flow chart of the two-scale Newton-Raphson iterative is shown in Fig. 6. Note that based on (i) and (ii), when inelasticity appears on both scales, each global iteration induces several iterations at local level. In this sense, global convergence implies local converge on each non-prismatic part.

Note also that inelasticity at local scale can induce iterations at global scale but the converse is not true. On the contrary, 


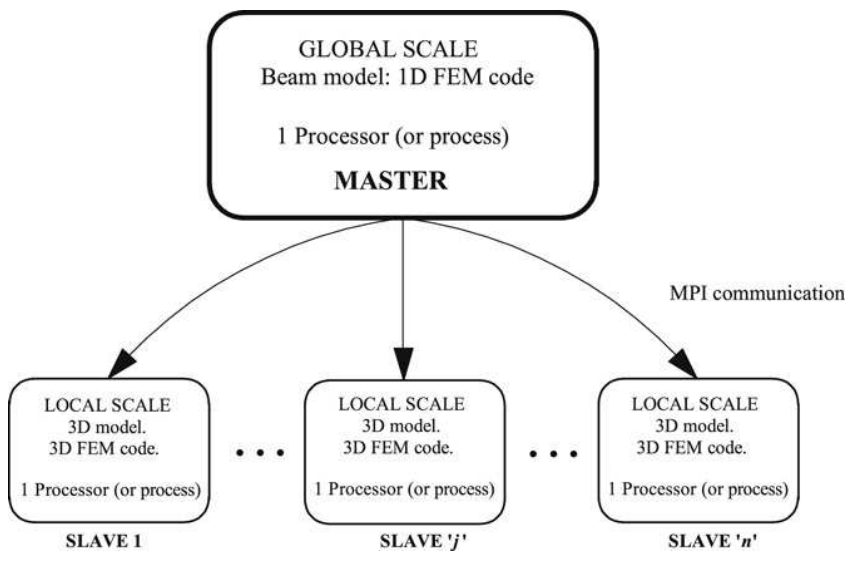

Fig. 7. Schematic representation of the master-slave approach.

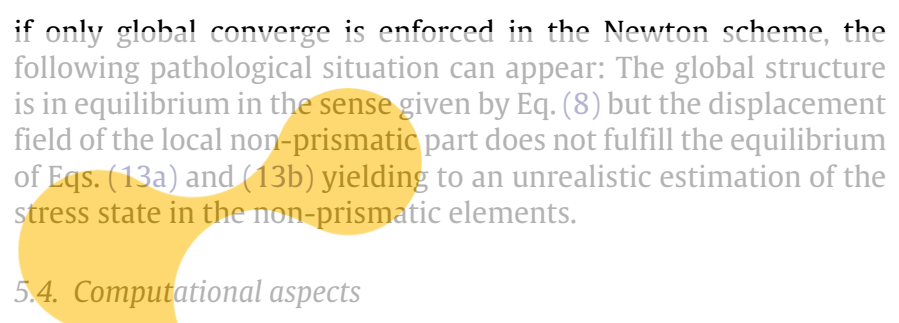

The proposed two-scale formulation for beam structures with local irregularities is very well suited to be computationally
implemented using the advantages of the parallel computation. The message passing interface (MPI) information among different process during execution. In the present case, a master-slave approach is of them, one of them, the master, is specific for the structural analysis of beams and the another one, corresponding to the slave, specific for

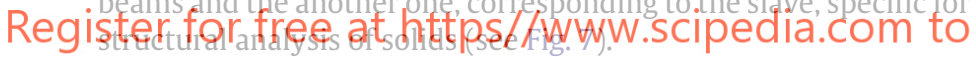
The following steps summarize the basic features of the computational implementation of the Newton-Raphson iterative scheme described in Section 5.3:

(i) The program which solves the global scale problem acts as master and initially sends the order to run, in independent processes, as many slave programs as local non-prismatic parts appear in the structure.

(ii) Each one of the slaves is an independent process corresponding to the execution of a specific program for the analysis of solids, which is in charge of solving the local scale problem. It is worth to note that each slave process can be executed on the same processor of the computer as the master or in a different one if a multiprocessor computer is being used. In the first case, the time required for the calculations increases linearly $^{9}$ with the number of slaves because the processor can run only one program at the same time. In the second case, the time required decreases because the structural response from several slaves can be obtained simultaneously. In any case, the use of the MPI system helps to perform a minimal intervention on existing codes specific for the analysis of 1D or 3D structures. The main advantage of using the MPI standard lies in the fact that the changes to be implemented in existing codes are

\footnotetext{
${ }^{9}$ Here the word 'linearly' is used to indicate that the time required for calculations is function of the number of slaves, since it would not be possible to maintain a strict linearity in the consumption of time when inelasticity has place in the models.
}

limited to include the SEND and RECEIVE calls corresponding to the synchronized message passing between the master and each one of the slaves.

(iii) The message passing among processes corresponds to the sending from the master of the rotation and displacement fields of the surface-interfaces of each slave and the receiving from the slaves to the master of the reduced forces, moments and tangential tensors corresponding to each iteration according to the flow chart of Fig. 6. Other information, typical of iterative schemes, such as global convergence in a time step or warnings are also passed for the opportune updating of the data base in the slave processes.

\subsection{Limitations of the present formulation and its computational implementation}

From the computational point of view, in spite of the mentioned advantages obtained from using the MPI system some difficulties can be mentioned:

(i) It is rather difficult to debug the execution of the coupled problem using the standard debugging tools of the modern developer environments which are, in most cases, developed for the construction of individual programs.

(ii) For the moment, there is not control over the order in which the synchronized messages are passed from different slaves to the master. This aspect is complicated when studying the

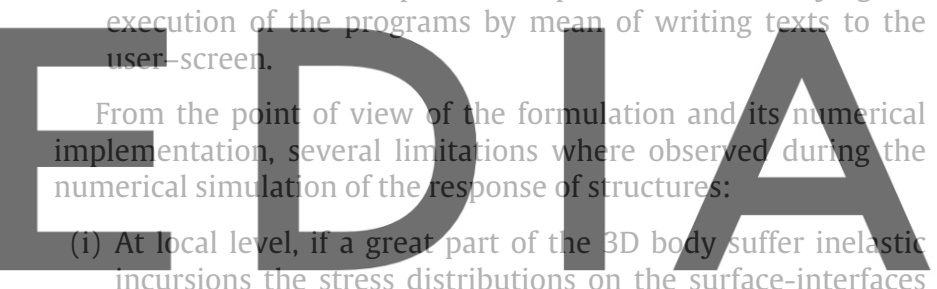

can become very complex influencing the global convergence.

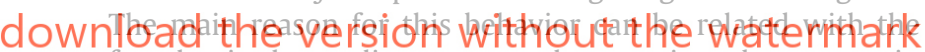
fact that in the nonlinear range, plane sections do not remain plane $^{10}$ and, therefore, the resulting reduced forces and moments have values that are far from the ones obtained on the cross sections of the connecting beams. A possible solution for this problem consists of considering local models representing a larger part of the structure, in such a way that the local inelastic effects are concentrated in a zone that remains far from the surface interfaces, limiting the inelasticity. By the other hand, if the proposed solution is employed, the massless assumption for the local scale model can introduce larger errors in the dynamic analysis and a new formulation considering inertial forces appears to be necessary.

It is worth to mention that normally when inelastic behavior appear in prismatic RC members, plane sections where strain localization occur loose their planarity. However, it is widely accepted that planarity (and a linear strain distribution) is recovered at a distance equal to the height of the cross section from the localized zone. Then, numerical models of local non-prismatic parts should locate the surfaceinterfaces at a distance from the zone where the geometry is complex no shorter than the largest dimension of the cross section of the connecting beams. This is the criterion used in the construction of the numerical models of the examples presented in the following section.

\footnotetext{
10 Mainly, when degrading materials are used.
} 


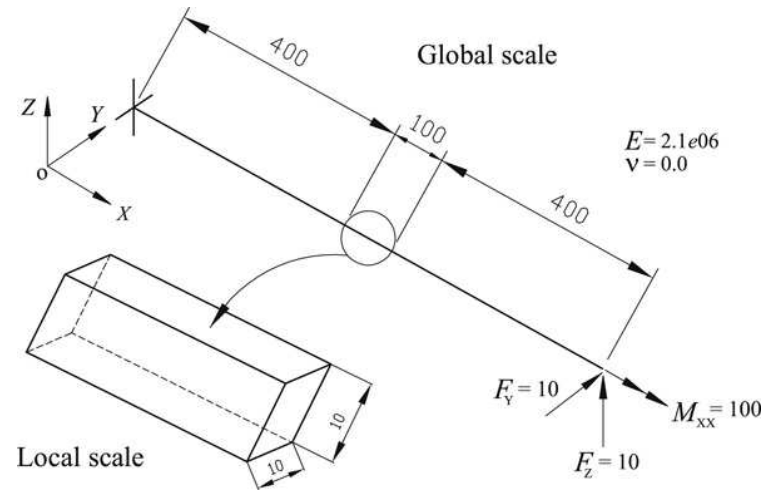

Fig. 8. Elastic cantilever beam subjected to 3D loading.

(ii) Imposing the restrictions given in Eq. (13b), enforces to maintain the size and shape of the $\partial \boldsymbol{\Omega}_{p s k}$; therefore, reaction forces contained in the plane of the surface-interfaces derived from the Poisson coefficient, $v$, of the materials become those forces (shear forces and torsion moment) ' $v$ dependent'. To overcome the difficulties encountered in the numerical simulations an additional assumption has been done, corresponding to assume $v=0$ for the materials of the FE's lying on the surface-interfaces. In this way, the plane and undeformed hypothesis assumed for beam elements is enforced in the local scale.

(iii) The number of local non-prismatic parts has to be defined prior to run the numerical simulations and, in this sense, the size of the problem is predefined. It would be desirable to have a specific criteria for deciding when a local model of a part of the structure has to be constructed. This last choice would allow save memory resources when not all the critical zones are in the nonlinear range.

\section{Numerical examples}

\subsection{Elastic example}

The fist example corresponds to the 3D loading of the cantilever beam of Fig. 8 . The beam is simulated using 5 quadratic beam elements for each of the segments located on the right and left sides of the central one. The central segment is simulated using a two-scale approach. Ten linear hexahedra are used in the direction $X$ and a mesh of $4 \times 4$ hexahedra are used for the surface interface, giving a total of 192 elements in the local model. One Gauss integration point is used in each FE of the local model. The applied loadings as well as the material properties are given in the same figure.

Fig. 9 shows a comparison between the displacement response in the $Y$ and $Z$ directions obtained from the two-scale model and the ones obtained using a simple elastic FE beam model. Its is possible to see a good agreement between both results.

Fig. 10 shows the distribution of the reaction forces on the surface-interface of the local model. It is worth noting that complex force distributions are well reproduced in the local model due to its capacity for representing the full 3D geometry of the beam, in contrast with that obtained in the beam model, due to the limitations of the kinematics assumptions. Moreover, as it has been explained before, reduced forces and moments are then calculated using Eq. (16).

Some additional comments can be made: (1) The example is solved using: (i) a two-scale model with 192 (linear) hexahedral elements and 10 quadratic beams, giving a total of 212 integration points; (ii) 11 quadratic elements (22 integration points) if only beam were used and (iii) 1728 elements (and integration points) if only hexahedra were used. The execution time for each loading step is: $2.40 \mathrm{~s}, 0.27 \mathrm{~s}$ and $17.8 \mathrm{~s}$ for cases (i), (ii) and (iii), respectively. It is possible to see the advantage of using the twoscale approach in the sense of reducing the execution time about 7.4 times when compared with the full 3D model. (2) The time delay in the communication between local and global scales is negligible due to the fact that parallelism is established between processes belonging to the same processor (AMD Athlon (tm) $64 \times$ 2 Dual. Core processor $4400+, 3$ GB RAM). However, for a problem including several local models solved in different processors, time delay spent in communication may become significant.

\subsection{Nonlinear static example}

The seismic response of the precast RC industrial building of Fig. 11 has been studied by the authors in reference [37] considering monolithic joints. The building has a bay width of $24 \mathrm{~m}$ and $12 \mathrm{~m}$ of inter-axes length. The story hight is $12 \mathrm{~m}$. The concrete has a ultimate compression of $35 \mathrm{MPa}$, with $E=290.000 \mathrm{MPa}, v=$ 0.2 and a tension/compression relation of 10 . The ultimate tensile stress for the steel is $510 \mathrm{MPa}$ with $v=0.15, E=200000 \mathrm{Mpa}$ and $v=0.15$. The dimensions of the columns are $60 \times 60 \mathrm{~cm}^{2}$. The beam has an initial high of $40 \mathrm{~cm}$ on the supports and $140 \mathrm{~cm}$ in the middle of the span. The permanent loads are $1000 \mathrm{~N} / \mathrm{m}^{2}$ and the weight of upper half part of the closing walls with $225.000 \mathrm{~N}$.

The building is meshed using 8 quadratic beam elements with two Gauss integration points for the resulting beams and column.
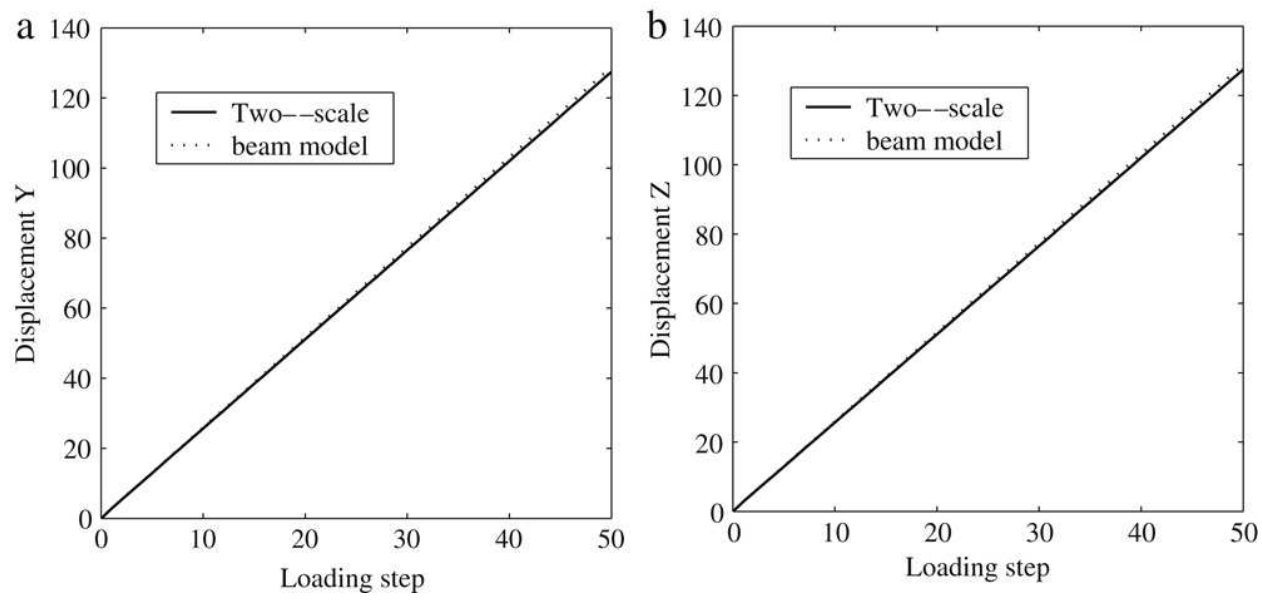

Fig. 9. Comparison between the displacement response obtained from the beam and the two-scale models ( $a, b)$ : Displacements in $Y$ and $Z$ directions, respectively. 
a

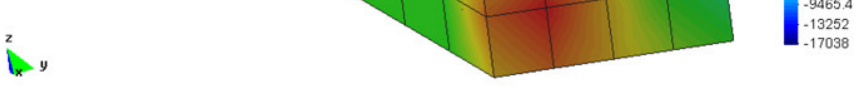

$\mathrm{b}$

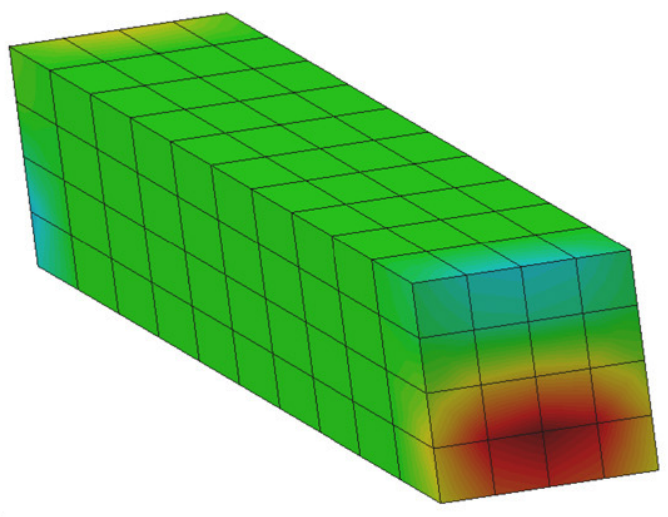

c
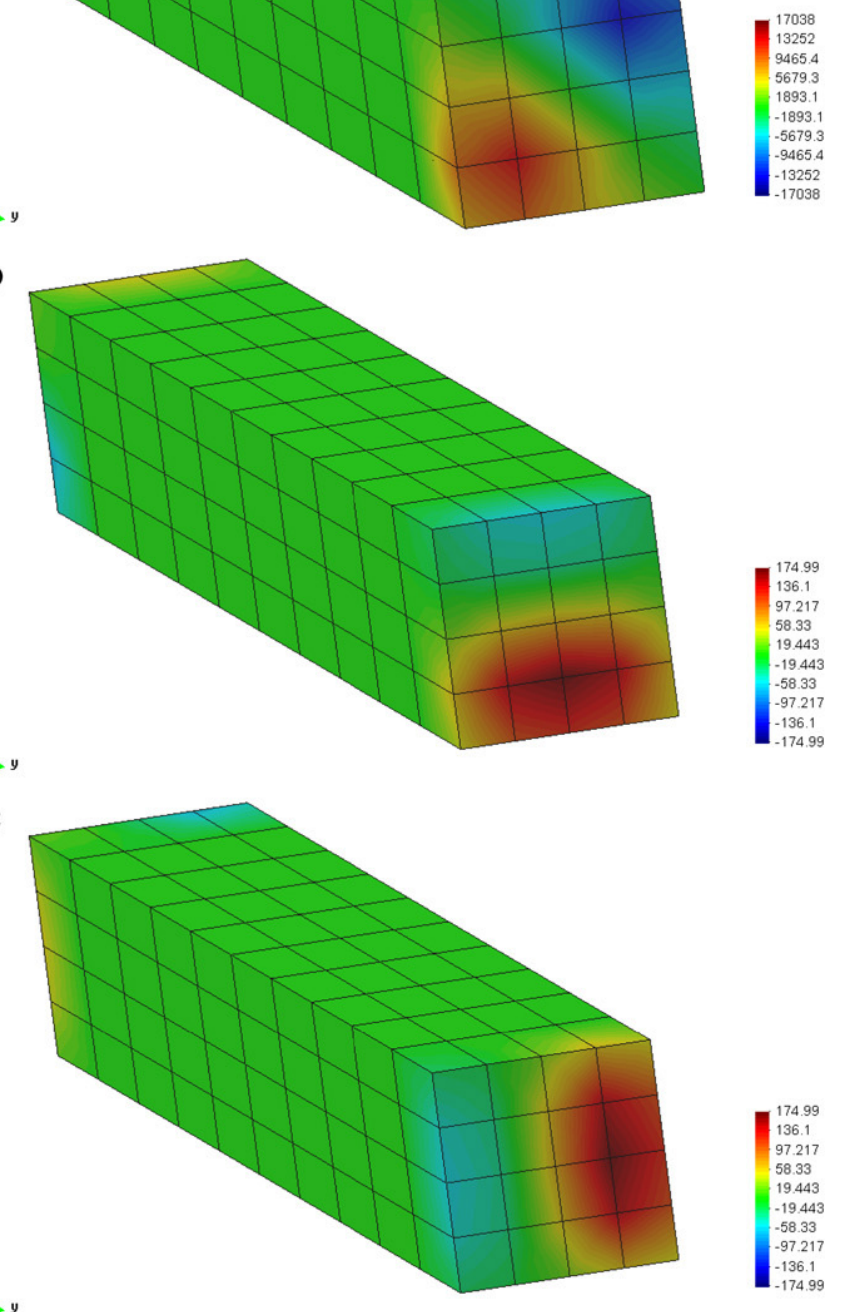

Fig. 10. Reaction force distributions on the surface-interfaces. (a): Force in $X$ direction. (b): Force in $Y$ direction. (c): Force in $Z$ direction. a

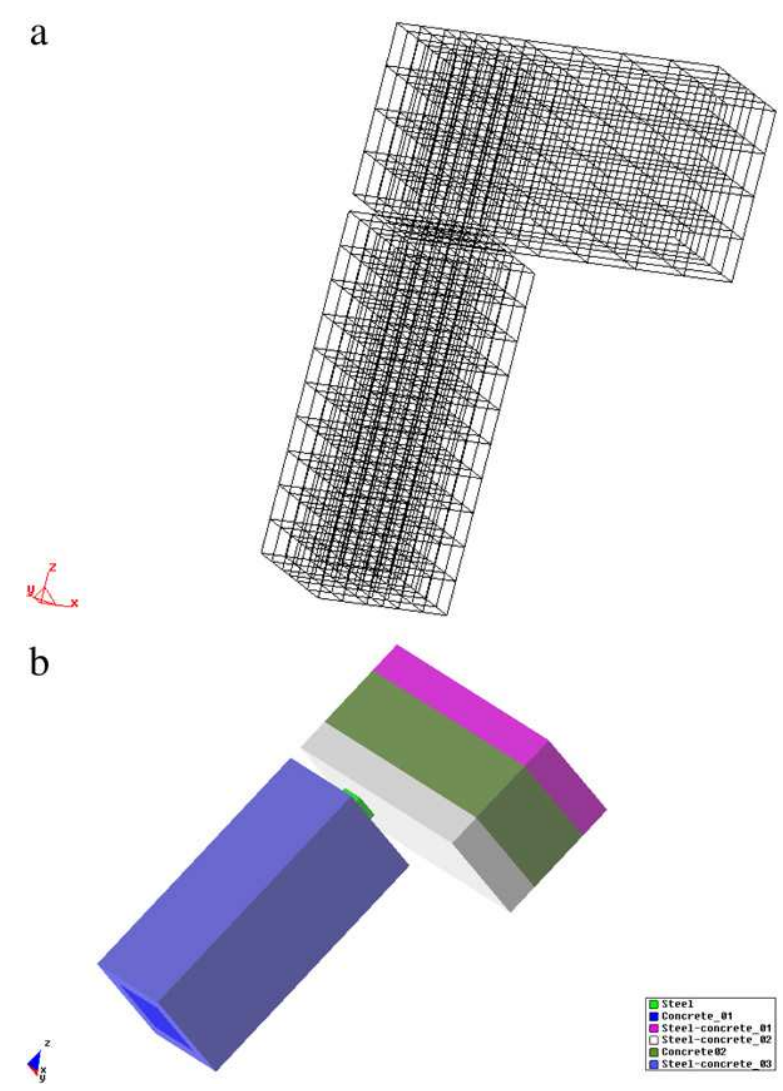

Fig. 12. FE model of the local scale problem. (a): Mesh. (b): Distribution of materials.

Details about the steel reinforcement and the grid of quadrilateral of the cross sections can be consulted in the same reference. Additionally, we consider a local scale model corresponding to the precast connecting joint which is clearly a local irregularity in the structure, see Fig. 11. The FE model used for the local scale is shown in Fig. 12 and it corresponds to a segment of the column and beam of $60 \mathrm{~cm}$ measured along their respective axis, which allows to cover completely the connecting zone where two steel bars with $16 \mathrm{~mm}$ diameter are used to transmit forces and moments among vertical and horizontal elements.

A total of 608 hexahedral FE are used with one Gauss integration point. Considering that the mixing rule is used in the local 3D model, several zones (see Fig. 12b) with different volumetric fractions of steel and concrete are defined, representing the longitudinal and transversal reinforcements in the structure. A static pushover analysis is performed considering: (i) the frame with monolithic joints in finite deformation. (ii) The two-scale

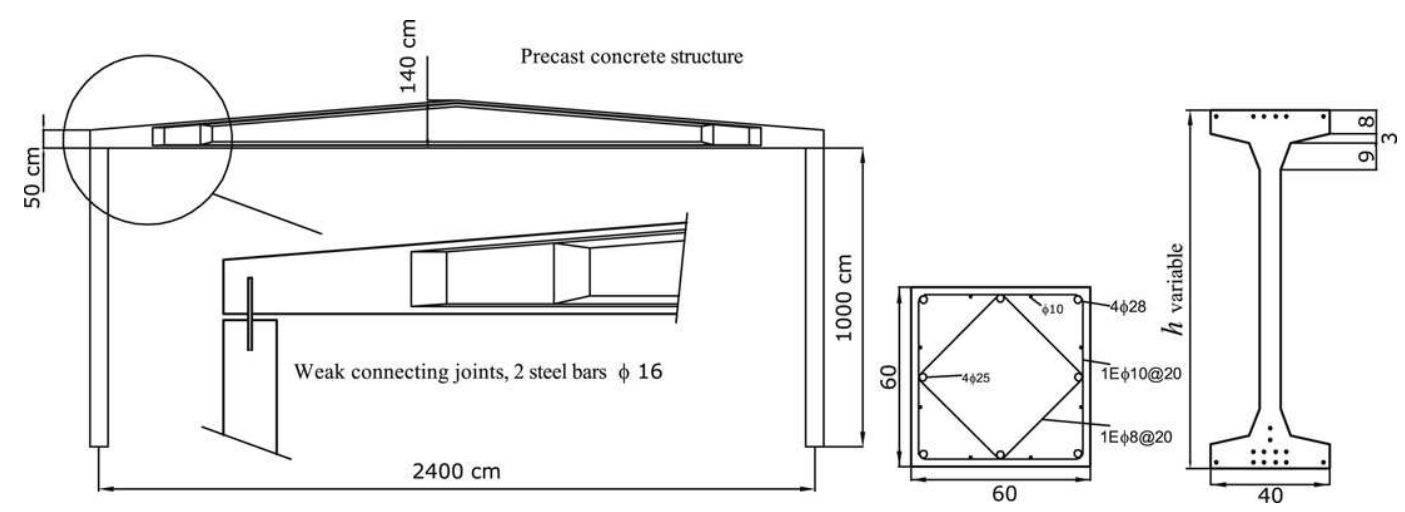

Fig. 11. Description of the structure. 

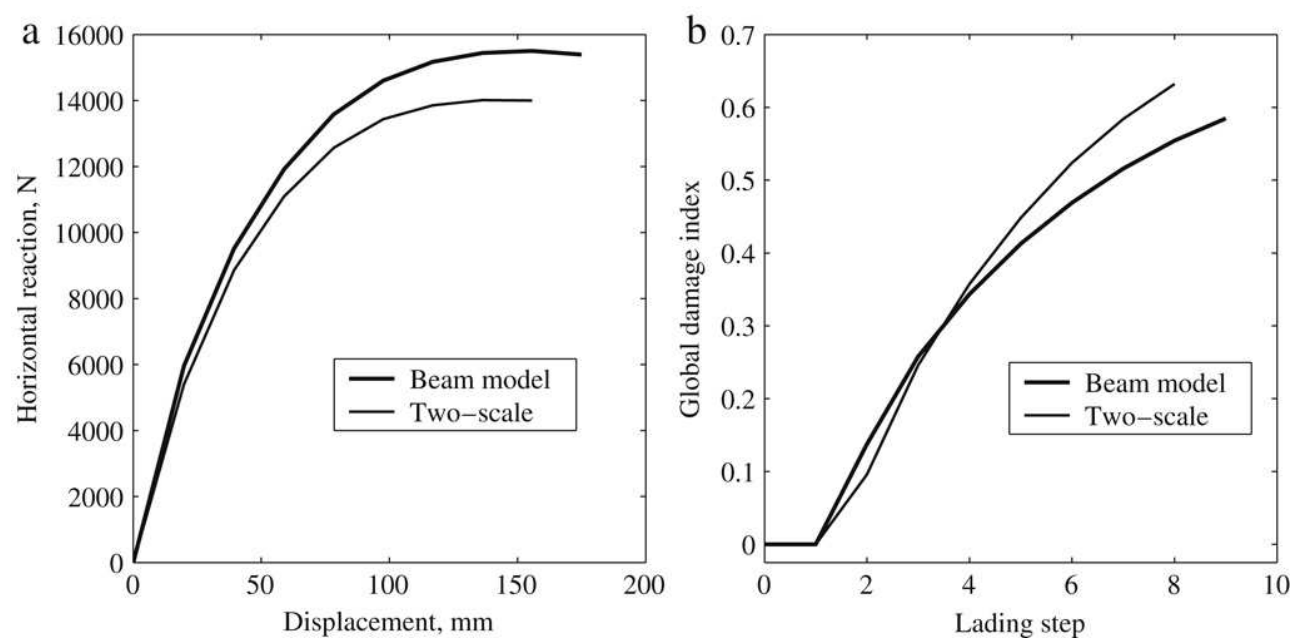

Fig. 13. (a): Capacity curves. (b): Evolution of the global damage index.

model in finite deformation. The purpose is to establish clearly the importance of considering an appropriated description of the local irregularities in the structures as well as the second order effect coupled with inelasticity in the study of flexible structures.

Fig. 13a shows the capacity curves obtained for the two mentioned cases. It is possible to see in this figure that the stiffness and ductility levels are overestimated in the case when the monolithic joints are employed. The use of finite deformation allows to take into account the so called effect $P-\Delta$ [37] and an anticipated strength degradation is observed for displacements over $60 \mathrm{~mm}$ which is a lateral displacement level expectable under strong seismic excitations. Softening behavior is observed for both models. Fig. 13b shows the evolution of the global damage index for both models. This index grows quickly for the two-scale model due to the fact that the greater part of the degradation and energy dissipation take place inside of the connecting joint.

\subsection{Nonlinear dynamic example}

In this example, the nonlinear seismic response of the structure of Section 6.2 is simulated by means of the two-scale model. The input acceleration is the $\mathrm{N}-\mathrm{S}$ component of the El Centro 1940 earthquake record. The response obtained from the two-scale model is compared with that obtained from the nonlinear dynamic response of a beam model. Fig. 14 shows the hysteretic cycles obtained from the lateral displacement of the upper beam-column joint and the horizontal reaction (base shear) in the columns for both models. Again it is possible to appreciate the influence of the local irregularity in the dynamic response: more energy dissipation and lateral displacements are obtained in the two-scale model.

Fig. 15 shows the time history response of the horizontal displacement and acceleration of the upper beam-column joint for both models. Displacements and accelerations are greater $(\sim 45 \%$ and $\sim 14 \%$, respectively) when the two-scale model is used in the numerical simulations, revealing that the nonlinear seismic response of precast and of other RC structures with local irregularities can be dominated by the mechanical behavior of the joints.

Fig. 16 shows several stages of the nonlinear inclusions experienced by the local model.

Finally, some additional comments: In Sections 6.2 and 6.3, most of the computing time was consumed in the local scale calculations (1824 DOFs versus 21 DOF in the global model). Moreover, the number of iterations and the time consumption in each iteration increased when the structure presented larger incursions in the nonlinear range. Typical values at the end of the simulations were: (i) Iterative computing time (a) Global scale:

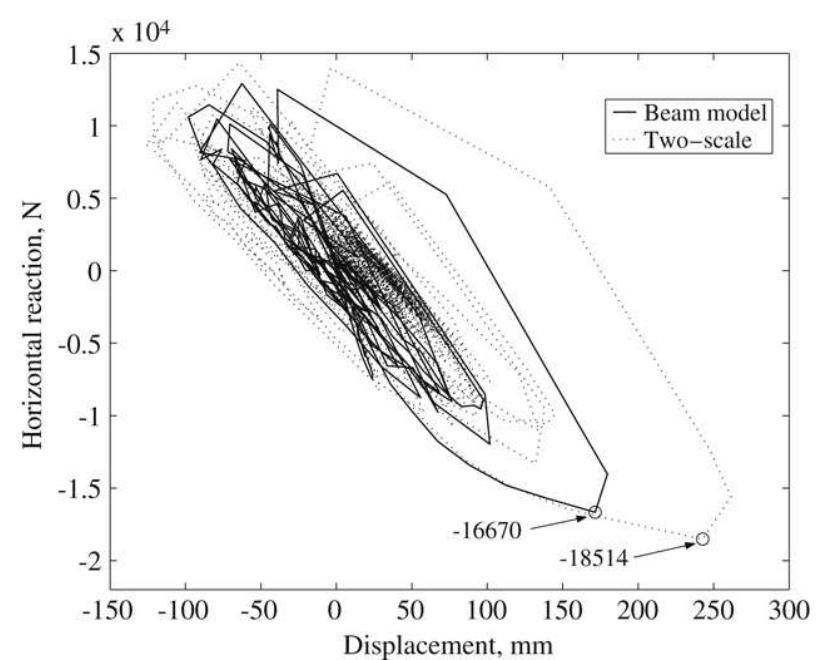

Fig. 14. Base shear-displacement relationship.

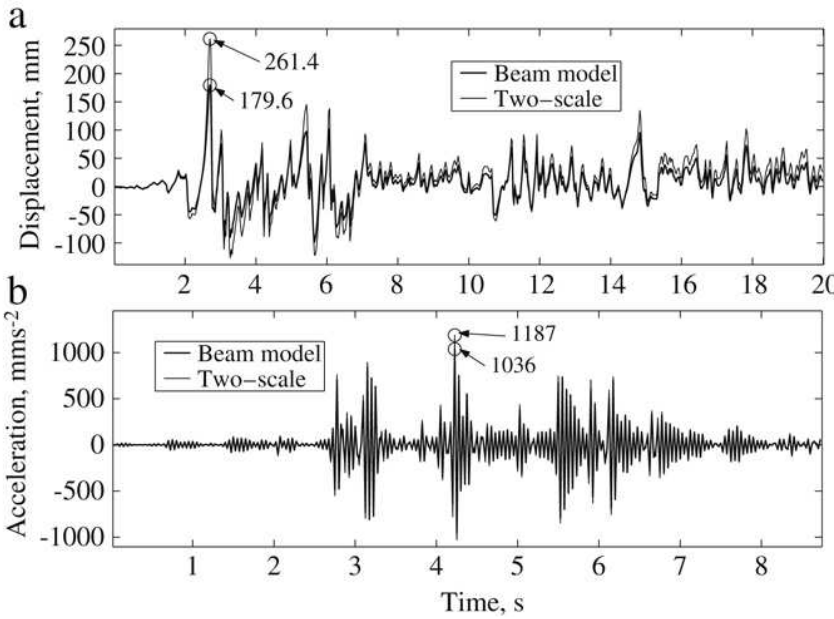

Fig. 15. Time history responses of the top beam-column joint. (a): Horizontal displacement. (b): Acceleration.

$\sim 25 \mathrm{~s}$ (b) Local scale: $\sim 5.5 \mathrm{~min}$. (ii) Number of iterations to converge: (a) Global scale: $\sim 20-35$. (b) Local scale: $\sim 30-50$.

Again, time delay in the communication process between local and global problems is negligible by the same reasons as explained in Section 6.1. The numerical experiments indicate that more 


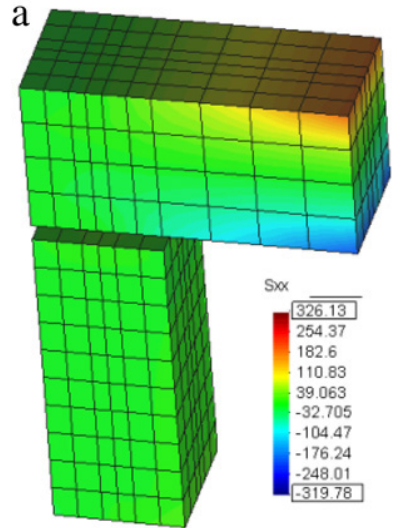

$t=2.0$

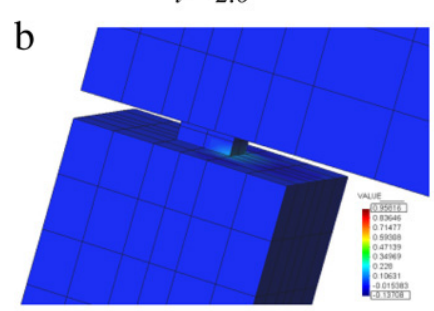

$t=2.0$

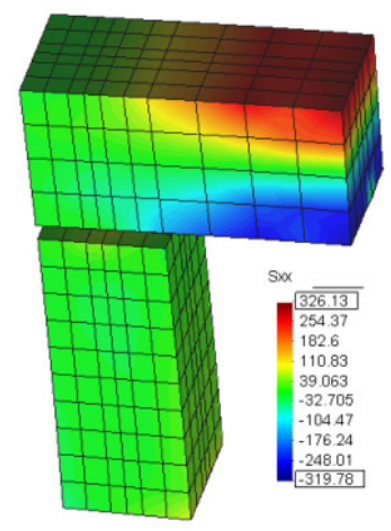

$t=3.1$

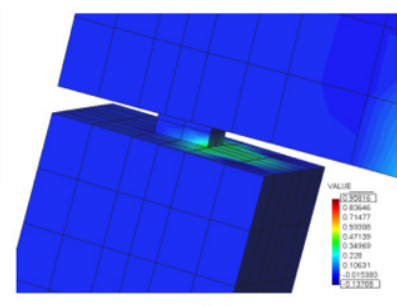

$t=3.1$

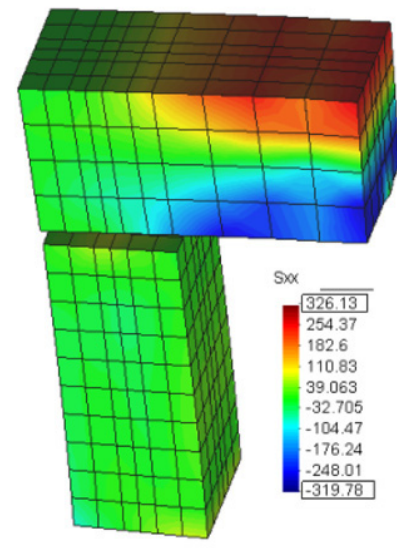

$t=4.2$

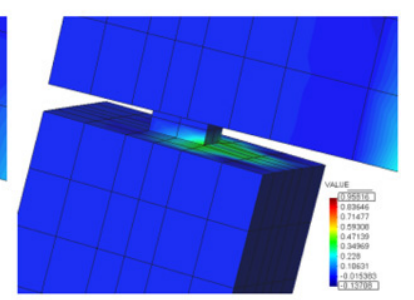

$t=4.2$

Fig. 16. Nonlinear response in the local model at times $t=2.0,3.1,4.2 \mathrm{~s}$. (a): Stress in the $Y$ direction. (b): Damage concentration around the connecting bar in the joint.

pronounced difficulties to achieve convergence appear according to the size of the part of the structure that suffer inelastic incursions.

\section{Conclusions}

A two-scale, global and local, approach has been developed for studying the nonlinear response of RC framed buildings which present local irregularities. At global scale level, all the elements of the model are 1D i.e. prismatic rods which consider the geometrically exact formulation due to Reissner and Simo, extended to include an arbitrary distribution of inelastic composite materials in the cross sections. Constitutive laws for the simple materials are based on thermodynamically consistent formulations obtaining more realistic estimations of the dissipated energy. A meshindependent response is obtained by means of the regularization of the energy dissipated at constitutive level. The simple mixing rule is applied for composites. An appropriated cross-sectional analysis is used to treat inelasticity at cross-sectional level. Consistent tangential constitutive tensors are considered to obtain the tangential beam stiffness matrix by means of cross-sectional integration.

If local geometric irregularities appear, a refined model of the corresponding element is carried out, constructing a fully 3D model which constitutes the local scale level. In analogy with the rods, inelastic composite materials are considered. The dimensionalcoupling between scales is developed through surface-interfaces imposing the kinematic hypothesis assumed for the beam model which consider finite deformation. Starting from the full 3D stress state existing in the local model, cross-sectional forces and moments, required at global level, are recovered by integrating the reaction forces at surface-interface level.

An iterative Newton-Raphson scheme based on the displacement method, which considers the interaction between scales is developed to obtain the response at the global level even in the nonlinear dynamic range. Equilibrium is checked at both, local and global levels, ensuring that compatible configurations are reached for the whole problem. The tangential stiffness of the local model is obtained numerically applying the perturbation method on the current configuration and obtaining the corresponding reaction forces reduced to the degree of freedom of the rod model.

The computational implementation manages the problem by means of the master-slave approach. The global scale problem acts as the master, sending the iterative displacement/rotation fields to the local scale models which are the (slaves) and then receives the corresponding internal forces, moments and tangential tensors obtained by integration on the surface-interfaces. The iterative process is stopped when the global convergence is achieved. The proposed approach is implemented in a parallelized algorithm, where the mater and slave problems are solved independently by different programs. The message passing between processes and/or processors is carried out using the MPI library of communication. In this way, minimal intervention on specific codes for beams and solids is required.

Additionally, the presented framework for the treatment of local geometric irregularities can be easily extended to include other type of kinematic assumptions for the beam model, modifying appropriately the restrictions imposed on the displacements of the surface-interfaces. Moreover, the constitutive models used in both scales can be selected according to the degree of refinement required by the user and are not limited only to the cases discussed here.

Finally, numerical examples are included showing the capabilities of the proposed formulation in the elastic and inelastic dynamic cases, highlighting the effect of considering appropriately the effects of local irregularities. The present formulation appears as a convenient approach for studying the dynamic nonlinear behavior or realistic RC structures where the response of the structure is dominated by local irregularities such as is the case of precast structures. The employment of refined FE models only at local level combined with a parallelizing computational techniques minimize the size of the problem allowing the application of advanced numerical techniques to realistic structures of engineering.

\section{Acknowledgments}

This research was partially supported by by the Spanish Government: Ministerio de Fomento and Ministerio de Ciencia 
y Tecnología. Project C 21/2006, Retrofitting and reinforcement of reinforced concrete structures with composite materials; Ministerio de Educación y Ciencia. Project BIA2005-06952, Study of composite materials for design, reinforcement and retrofit of civil engineering structures (RECOMP). Project PSE 11-2005 (PSS-380000-200514) (HABITAT 2030). Project CSD2006-00060 SEDUREC, Seguridad $y$ durabilidad de estructuras de construcción. The International Center for Numerical Methods in Engineering (CIMNE) has also provided the support for this research. All this support is gratefully acknowledged.

\section{References}

[1] Aristizabal-Ochoa JD. Large deflection and postbuckling behavior of Timoshenko beam-columns with semi-rigid connections including shear and axial effects. Eng Struct 2007;29:991-1003.

[2] Armero F, Ehrlich D. Numerical modeling of softening hinges in the Euler-Bernoulli beams. Comput Struct 2005;84:641-56.

[3] Barbat AH, Oller S, Hanganu A, Oñate E. Viscous damage model for Timoshenko beam structures. Int J Solids Struct 1997;34:3953-76.

[4] Bathe KJ. Finite element procedures. Prentice Hall, Inc.; 1996.

[5] Bayrak O, Sheikh SA. Plastic hinge analysis. J Struct Eng 2001;127:1092-100.

[6] Benavent-Climent A. Shaking table tests of reinforced concrete wide beam-column connections. Earthq Eng Struct Dyn 2005;34:1833-9.

[7] Bratina S, Saje M, Planinc I. On materially and geometrically non-linear analysis of reinforced concrete planar frames. Int J Solids Struct 2004;41: 7181-207.

[8] Cardona A, Gerardin M. A beam finite element non-linear theory with finite rotations. Int J Numer Methods Eng 1988;26:2403-38.

[9] Car E, Oller S, Oñate E. An anisotropic elasto plastic constitutive model for large strain analysis of fiber reinforced composite materials. Comput Methods App Mech Eng 2000; 185:245-77.

[10] Cosenza E, Manfredi G, Verderame GM. A fibre model for push-over analysis of underdesigned reinforced concrete frames. Comput Struct 2006;84:904-16.

[11] Crisfield MA. Non-linear finite element analysis of solids and structures, (vols. 1 \& 2). John Wiley \& Sons; 1998

[12] Davenne L, Ragueneau F, Mazars J, Ibrahimbegović A. Efficient approaches to finite element analysis in earthquake engineering. Comput Struct 2003;81: 1223-39.

[13] Faleiro Freitas J, Oller S, Barbat AH. Plastic-damage seismic model for reinforced concrete frames. Comput Struct 2008;86:581-97.

[14] Fu F, Lam D, Ye J. Parametric study of semi-rigid composite connections with 3-D finite element approach. Eng Struct 2007;29:888-98.

[15] Garusi E, Tralli A. A hybrid stress-assumed transition element for solid-tobeam and plate-to-beam connections. Comput Struct 2002;80:105-15.

[16] Gopalakrishnan S, Doyle JF. Spectral super-elements for wave propagation in structures with local non-uniformities. Comput Methods Appl Mech Eng 1995; 121:77-90.

[17] Gruttmann F, Sauer R, Wagner W. Theory and numerics of three-dimensional beams with elastoplastic material behavior. Int J Numer Methods Eng 2000 48:1675-702.

[18] Hailu D, Zekaria A, Kassegne AK. A new efficient multiple-node constraint approach for FEA analysis of radius-cut RBS moment frames in highly seismic areas. In: Proceedings of the joint EACE-AAU international conference on computational mechanics. Structures and earthquake engineering. 2003.

[19] Halliday PJ, Grosh K. Dynamic response of complex structural intersections using hybrid methods. J Appl Mech 1999;66:653-9.

[20] Hanganu AD, Oñate E, Barbat AH. Finite element methodology for local/global damage evaluation in civil engineering structures. Comput Struct 2002;80: 1667-87.

[21] Hajjar JF, Molodan A. A distributed plasticity model for cyclic analysis of concrete-filled steel tube beam-columns and composite frames. Eng Struct 1998;20:398-412.

[22] Iura M, Atluri SN. On a consistent theory, and variational formulation of finitely stretched and rotated 3-D space-curved beams. Comput Mech 1989;4:73-88.

[23] Ibrahimbegović A. On finite element implementation of geometrically nonlinear Reissner`s beam theory: Three-dimensional curved beam elements. Comput Methods Appl Mech Eng 1995;122:11-26.

[24] Izzuddin BA, Lloyd Smith D. Efficient nonlinear analysis of elasto-plastic 3D $\mathrm{R} / \mathrm{C}$ frames using adaptive techniques. Comput Struct 2000;78:549-73.

[25] Jelenic̀ G, Crisfield MA. Geometrically exact 3D beam theory: Implementation of a strain-invariant finite element for static and dynamics. Comput Methods Appl Mech Eng 1999;171:141-71.

[26] Jelenic̀ G, Crisfield MA. Dynamic analysis of 3D beams with joints in presence of large rotations. Comput Methods Appl Mech Eng 2001;190:4195-230.

[27] Kapania RK, Li J. On a geometrically exact curved/twisted beam theory under rigid cross-section assumption. Comput Mech 2003;30:428-43.

[28] Kapania RK, Li J. A formulation and implementation of geometrically exact curved beam elements incorporating finite strains and finite rotations. Comput Mech 2003;30:444-59.

[29] Lee SS, Moon TS. Moment-rotation model of semi-rigid connections with angles. Eng Struct 2002;24:227-37.
[30] Lubliner J, Oliver J, Oller S, Oñate E. A plastic-damage model for concrete. Int J Solids Struct 1989;25:299-326.

[31] Luccioni B, Oller S, Danesi R. Coupled plastic-damaged model. Comput Methods Appl Mech Eng 1996;129:81-9.

[32] Mäkinen J, Marjamäki H. Total Lagrangian Parametrization of rotation manifold. In: Fifth EUROMECH nonlinear dynamics conference. 2005. p. 522-30.

[33] Marsden JE, Hughes TJR. Mathematical foundations of elasticity. California: Prentice-Hall; 1983.

[34] Martinez X, Oller S, Rastellini F, Barbat AH. Numerical procedure for the computation of RC structures reinforced with FRP using the serial/parallel mixing theory. Comput Struct 2008;86:1604-18.

[35] Mata P, Oller S, Barbat AH. Static analysis of beam structures under nonlinear geometric and constitutive behavior. Comput Methods Appl Mech Eng 2007; 196:4458-78

[36] Mata P, Oller S, Barbat AH. Dynamic analysis of beam structures considering geometric and constitutive nonlinearity. Comput Methods Appl Mech Eng 2007; $197: 857-78$

[37] Mata P, Barbat AH, Oller S, Boroschek K. Nonlinear seismic analysis of RC structures with energy dissipating devices. Int Numer Methods Eng 2007 [submitted for publication]

[38] McCune RW, Armstrong CG, Robinson DJ. Mixed-dimensional coupling in finite element models. Int J Numer Methods Eng 2000;49:725-50.

[39] Monaghan DJ, Lee KY, Armstrong CG, Ou H. Mixed dimensional finite element analysis of frame models. In: Proc. 10th ISOPE conference, vol. 4. 2000. p. 263-69.

[40] Neuenhofer A, Filippou FC. Evaluation of the nonlinear frame finite-element models. J Struct Eng 1997; 123:958-66.

[41] Oliver J, Cervera M, Oller S, Lubliner J. Isotropic damage models and smeared crack analysis of concrete. In: Proceedings 2nd ICCAADCS, 2. Zell Am See, Austria: Pineridge Press; 1990. p. 945-58.

[42] Oliver J, Linero DL, Huespe AE, Manzoli OL. Two-dimensional modeling of material failure in reinforced concrete by means of a continuum strong discontinuity approach. Comput Methods Appl Mech Eng 2008;197:332-48.

[43] Oller S, Oñate E, Miquel J, Botello S. A plastic damage constitutive model for composites materials. Int J Solids Struct 1996;33:2501-18.

[44] Oller S, Oñate E, Miquel J. Mixing anisotropic formulation for the analysis of composites. Commun Numer Methods Eng 1996;12:471-82.

[45] Oller S, Canet JM, Zalamea F. Composite material behavior using a homogenization double scale method. J Eng Mech 2005;131:65-79.

[46] Oller S, Luccioni B, Barbat AH. Un método de evaluación del daño sísmico en pórticos de hormigón armado. Rev int de métodos numér para cálc y diseñ en ingenier 1996;12:215-38.

[47] Pampanin S. Alternative design philosophies and seismic response of precast concrete buildings. Struct Concrete 2003;4:203-11

[48] Pampanin S, Amaris A, Akguzel U, Palermo A. Experimental investigations on high-performance jointed ductile connections for precast frames. In: First European conference on earthquake engineering and seismology 2006. (Paper $\mathrm{N}: 2038)$.

[49] Papaioannou I, Fragiadakis M, Papadrakakis M. Inelastic analysis of framed structures using the fiber approach. In: Proceedings of the 5th international congress on computational mechanics. 2005. p. 231-38.

[50] Reissner E. On one-dimensional large-displacement finite-strain beam theory. Stud Appl Math 1973;11:87-95.

[51] Reissner E. On one-dimensional finite-strain beam theory: The plane problem. J Appl Math Phys 1972;23:795-804.

[52] Saje M, Turk G, Kalagasidu A, Vratanar B. A kinematically exact finite element formulation of elastic-plastic curved beams. Comput Struct 1998:67:197-214.

[53] Shao Y, Aval S, Mirmiran A. Fiber-element model for cyclic analysis of concrete-filled fiber reinforced polymer tubes. J Struct Eng 2005;131:292-303.

[54] Shim KW, Monaghan DJ, Armstrong CG. Mixed dimensional coupling in finite element stress analysis. Eng Comput 2002;18:241-52.

[55] Shim KW, Monaghan DJ, Armstrong CG. Mixed dimensional coupling in finite element stress analysis. In: 10th international meshing roundtable, Sandia National Laboratories. 2001. p. 269-77.

[56] Simo JC. A finite strain beam formulation. The three-dimensional dynamic problem. Part I. Comput Methods Appl Mech Eng 1985;49:55-70.

[57] Simo JC, Vu-Quoc L. A three-dimensional finite-strain rod model. Part II Computational aspects. Comput Methods Appl Mech Eng 1986;58:79-116.

[58] Simo JC, Vu-Quoc L. On the dynamics in space of rods undergoing large motions-A geometrically exact approach. Comput Methods Appl Mech Eng 1988;66:125-61.

[59] Simo JC, Hjelmstad KD, Taylor RL. Numerical simulations of elasto-viscoplastic response of beams accounting for the effect of shear. Comput Methods App Mech Eng 1984;42:301-30.

[60] Snir M, Otto S, Huss-Lederman S, Walker D, Dongarra J. MPI The complete reference volume 1 . The MPI core. second edn. Massachusetts Institute of Technology; 1998.

[61] Spacone E, El-Tawil S. Nonlinear analysis of steel-concrete composite structures: State of the art. J Struct Eng 2000;126:159-68.

[62] Taucer FF, Spacone E, Filipou FC. A fiber beam-column element for seismic response analysis of reinforced concrete structures. Technical report No. UCB/EERC-91/17. Earthquake Engineering Research Center, University of California at Berkeley; 1991.

[63] Wenzel M. Consistent transition of beam-shell structures within the FE method. PAMM Proc in Appl Math \& Mech 2003;3:320-1.

[64] Ziyaeifara M, Noguchi H. A refined model for beam elements and beam-column joints. Comput Struct 2000;76:551-64. 